\title{
Blood flukes of Asiatic softshell turtles: revision of Coeuritrema Mehra, 1933 (Digenea: Schistosomatoidea) and a new species infecting Chinese softshell turtles, Pelodiscus sinensis (Trionychidae), from Vietnam
}

\author{
Jackson R. Roberts ${ }^{1}$, Raphael Orélis-Ribeiro ${ }^{1}$, Binh T. Dang ${ }^{2}$, Kenneth M. Halanych ${ }^{3}$ and Stephen A. Bullard ${ }^{1}$ \\ ${ }^{1}$ Auburn University, School of Fisheries, Aquaculture \& Aquatic Sciences and Aquatic Parasitology Laboratory, Auburn, AL, USA; \\ ${ }^{2}$ Nha Trang University, Department of Biological Sciences, Institute for Biotechnology and Environment, Nha Trang, Vietnam; \\ ${ }^{3}$ Auburn University, Department of Biological Sciences and Molette Biology Laboratory for Environmental \& Climate Change \\ Studies, Auburn, AL, USA
}

\begin{abstract}
Coeuritrema Mehra, 1933, previously regarded as a junior subjective synonym of Hapalorhynchus Stunkard, 1922, herein is revised to include Coeuritrema lyssimus Mehra, 1933 (type species), Coeuritrema rugatus (Brooks et Sullivan, 1981) comb. n., and Coeuritrema platti Roberts et Bullard sp. $\mathrm{n}$. These genera are morphologically similar by having a ventral sucker, non-fused caeca, two testes, a pre-testicular cirrus sac, an intertesticular ovary, and a common genital pore that opens dorsally and in the sinistral half of the body. Phylogenetic analysis of the D1-D3 domains of the nuclear large subunit ribosomal DNA (28S) suggested that Coeuritrema and Hapalorhynchus share a recent common ancestor. Coeuritrema is morphologically most easily differentiated from Hapalorhynchus by having ventrolateral tegumental papillae and a definitive metraterm that is approximately $3-7 \times$ longer than the uterus. Coeuritrema comprises species that reportedly infect Asiatic softshell turtles (Testudines: Trionychidae) only, whereas Hapalorhynchus (as currently defined) comprises blood flukes that reportedly infect those hosts plus North American musk turtles (Sternotherus Bell in Gray) and mud turtles (Kinosternon Spix), both Kinosternidae, North American snapping turtles (Chelydridae), Asiatic hard-shelled turtles (Geoemydidae) and African pleurodirans (Pelomedusidae). Coeuritrema platti sp. n. infects the blood of Chinese softshell turtles, Pelodiscus sinensis (Wiegmann), cultured in the Da Rang River Basin (Phu Yen Province, Vietnam). It differs from C. lyssimus by having a narrow hindbody $(<1.6 \times$ forebody width), ventrolateral tegumental papillae restricted to the hindbody, a short cirrus sac $(<10 \%$ of corresponding body length), a transverse ovary buttressing the caeca, a short, wholly pre-ovarian metraterm ( $\sim 10 \%$ of corresponding body length), and a submarginal genital pore. It differs from C. rugatus by having small ventrolateral tegumental papillae, testes without deep lobes, and a Laurer's canal pore that opens posterior to the vitelline reservoir and dorsal to the oviducal seminal receptacle. The new species is only the second turtle blood fluke reported from Vietnam.
\end{abstract}

Keywords: taxonomy, Spirorchiidae, 28S rDNA, molecular phylogeny, Griphobilharzia

The turtle blood flukes (TBFs; paraphyletic 'Spirorchiidae') comprise 84 accepted species (54 freshwater; 30 marine) assigned to 20 genera and that infect 37 freshwater and three marine turtle species (Platt 1993, 2002, Smith 1997a,b, Tkach et al. 2009, Platt and Sharma 2012, Orélis-Ribeiro et al. 2014, Roberts et al. 2016). Seventeen TBFs of five genera (Hapalorhynchus Stunkard, 1922; Vasotrema Stunkard, 1926; Coeuritrema Mehra, 1933; Enterohaematotrema Mehra, 1940, Cardiotrema Dwivedi, 1967) infect softshell turtles (Testudines: Trionychidae) (Table 1), but only nine of 31 (29\%) trionychids host a TBF, suggesting that perhaps numerous innominate TBFs infect this turtle lineage. Softshell turtles of Pelodiscus Fitzinger (P. axenaria [Zhou, Zhang et Fang], P. maackii [Brandt],
P. parviformis Tang, P. sinensis [Wiegmann]) are commercially prized and cultured for food and magic throughout Asia (Alves et al. 2008, Fritz et al. 2010, Stuckas and Fritz 2011). A survey of Chinese aquaculture facilities indicated that $>300$ million Chinese softshell turtles are in captivity now and that $\sim 125$ million individuals are available for sale now in China alone (Haitao et al. 2008). Even though they are abundant in southeast Asian aquaculture, several of these species have become scarce in the wild (van Dijk et al. 2000). Despite their commercial importance, the taxonomy of their blood parasites remains as underexplored as the infectious diseases that afflict these turtles in aquaculture. 
During a recent parasitological expedition to Vietnam, we encountered a new TBF species infecting Chinese softshell turtles, which led to a consideration of all TBFs infecting softshell turtles. Herein, we revise Coeuritrema, emend its diagnosis, redescribe Coeuritrema rugatus (Brooks et Sullivan, 1981) comb. n. (formerly Hapalorhynchus) based on museum specimens, describe a new species, and provide an updated phylogeny for the TBFs inclusive of the new taxon. This is the second TBF species reported from Vietnam.

\section{MATERIALS AND METHODS}

Turtles were purchased from commercial turtle trappers in Nha Trang, Vietnam and from a turtle farm in Phu Yen Province during 1-16 June 2015. Turtles were killed by decapitation. Necropsies were performed using $7.0 \mathrm{~g} / 1$ sodium citrate saline solution. Living flukes intended as whole-mounts were killed with a butane hand lighter under little or no coverslip pressure and transferred to a vial of $5 \%$ neutral buffered formalin (n.b.f.). TBFs were maintained in $5 \%$ n.b.f. until staining. After washing with distilled water, specimens intended as whole mounts were stained in Van Cleave's hematoxylin with several additional drops of Ehrlich's hematoxylin, dehydrated using an ethanol series, cleared in clove oil, and permanently mounted in Canada balsam.

Whole mounts were examined using a Leica DM 2500 microscope with differential interference contrast optical components. Parasite measurements are reported in micrometres $(\mu \mathrm{m})$ followed by the mean and number of specimens measured in parentheses. Turtle scientific names and taxonomic authorities follow van Dijk et al. (2014). Classification and anatomical terms for TBFs follow Luhman (1935 - genital sucker), Byrd (1939 - uterine pouch [see also Roberts et al. 2016]), Yamaguti (1971 - Manter's organ), and Platt (1993 - median oesophageal pouch; 1998, 2002 - genital spines, plicate organ and most other terms).

Specimens for molecular analyses were handled with camel-hair brushes, pipettes or fine forceps, and immediately preserved in a vial of absolute $\mathrm{EtOH}$ and stored at $-20^{\circ} \mathrm{C}$. Total genomic DNA (gDNA) was extracted using DNeasyTM Blood and Tissue Kit (Qiagen, Valencia, California, USA) according to the manufacturer's protocol, except for the incubation period with proteinase- $K$ that was extended to overnight, and the final elution step wherein only $100 \mu$ of elution buffer was used to increase the final DNA concentration in the eluate.

Partial 28S rDNA (domains D1-D3; 1400 bp) was amplified using the forward primer 'U178' (5'-GCACCCGCTGAAYTTAAG-3') and the reverse primer 'L1642' (5'-CCAGCGCCATCCATTTTCA-3') (Lockyer et al. 2003). PCR amplifications were performed using a total volume of $25 \mu \mathrm{l}$ containing approximately $2 \mu \mathrm{l}$ of DNA template, $0.4 \mu \mathrm{M}$ of each primer along with $1 \times$ buffer, $2.5 \mathrm{mM} \mathrm{MgCl}$ (New England Biolabs, Ipswich, Massachusetts, USA), $1 \mathrm{mM}$ dNTP mixture and $0.3 \mu 1$ Taq polymerase (5 U/ $\mu$ l) (New England Biolabs). Thermocycling profile consisted of an initial $4 \mathrm{~min}$ at $94^{\circ} \mathrm{C}$ for denaturation, followed by 40 repeating cycles of $94^{\circ} \mathrm{C}$ for $30 \mathrm{~s}$ for denaturation, $50^{\circ} \mathrm{C}$ for $30 \mathrm{~s}$ for annealing, and $72^{\circ} \mathrm{C}$ for $2 \mathrm{~min}$ for extension, followed by a final $5 \mathrm{~min}$ at $72^{\circ} \mathrm{C}$ for extension. All PCR reactions were carried out in a Veriti Thermal Cycler (Applied Biosystems, Waltham, Massachusetts, USA). PCR products $(5 \mu \mathrm{l})$ were verified on a $1 \%$ agarose gel and stained with ethidium bromide. PCR amplicons were gel-excised using QIAquickTM Gel Extraction Kit (Qiagen, Valencia, California, USA) following the manufacturer's protocol. DNA sequencing was performed by GENEWIZ with ABI Prism 3730xl DNA analyser (GENEWIZ, Inc., South Plainfield, New Jersey, USA). Primers used in sequencing of $28 \mathrm{~S}$ rDNA included the PCR primers and the internal forward primers 300F (5'-CAA GTACCGTGAGGGAAAGTTG-3') and 900F (5'-CCGTCTTGAAACACGGACCAAG-3') and reverse primer 1200R (5'-GCATAGTTCACCATCTTTCGG-3') (Lockyer et al. 2003). Sequence assembling and analysis of chromatograms were conducted using BioNumerics version 7.0 (Applied Maths, SintMartens-Latem, Belgium).

The sequence data for nuclear 28S rDNA generated during this study were aligned with those for selected blood flukes (Schistosomatoidea) available on GenBank. Outgroups were selected from representative fish blood flukes, Aporocotylidae Odhner, 1912 (see Bullard et al. 2009). The ingroup comprised newly-generated sequence data from the new species (KX712243) and Hapalorhynchus foliorchis Brooks et Mayes, 1975 (KX712242) plus publicly available sequences from Baracktrema obamai Roberts, Platt et Bullard, 2016 (KX061500), Griphobilharzia amoena Platt et Blair, 1991 (AY899914), and ten other TBFs (Orélis-Ribeiro et al. 2014). The specimens of H. foliorchis were collected in Alabama from Chelydra serpentina (Linnaeus) during October 2014 (Roberts et al. 2016). Sequences were aligned using MAFFT (Katoh and Toh 2010) with default settings implemented in the CIPRES Science Gateway V.3.3 (Miller et al. 2010). The resulting alignment was refined by eye using MEGA version 5.2.2 (Tamura et al. 2011) and the ends of each fragment were trimmed to match the shortest sequence. Ambiguous positions were identified and removed using the Gblocks server (Castresana 2000) with settings for a less stringent selection. Bayesian inference (BI) was performed using the Metropolis-coupled Markov chain Monte Carlo method (MC3) in MrBayes version 3.2.6 (Huelsenbeck et al. 2001, Ronquist and Huelsenbeck 2003, Huelsenbeck and Ronquist 2005) and run on CIPRES (Miller et al. 2010). The model of nucleotide substitution was selected based on the Akaike Information Criterion (Posada and Buckley 2004) as implemented in the jModelTest version 2.1.4 (Guindon and Gascuel 2003, Darriba et al. 2012). The GTR $+\mathrm{I}+\mathrm{G}$ (proportion of invariable sites $=0.385$ and gamma distribution $=1.393$ ) model was inferred as the best estimator. Therefore, BI used the following parameters: $n s t=6$, rates $=$ invgamma, ngammacat $=4$, and default priors. Analyses were run in duplicate each containing 4 independent chains (three heated and one cold chain) $($ nchains $=4)$ for $1.0 \times 107$ generations $($ ngen $=10000000)$ sampled at intervals of 1000 generations (samplefreq $=1000$ ). Results of the first 2500 sampled trees were discarded as 'burn-in' based on the stationarity of the likelihood values, assessed by plotting the log-likelihood values of the sample points against generation time using Tracer version 1.5 (Rambaut and Drummond 2009). All retained trees were used to estimate posterior probability of each node. A majority rule consensus tree with average branch lengths was constructed for the remaining trees using 'summarise the trees' (sumt) in MrBayes. Resulting phylogenetic trees were visualised using FigTree v1.3.1 (Rambaut 2009) and further edited with Adobe Illustrator CS3. Branch supports were considered as significant when posterior probabilities were $>0.95$. 


\section{RESULTS}

Coeuritrema Mehra, 1933 emended

Figs. 1-7

Diagnosis. Body dorsoventrally flattened (not cylindrical), 3-7× longer than wide, constricted at level of ventral sucker, having hindbody 1-3× longer than forebody, aspinous, papillate; ventrolateral tegumental papillae present, distributing from oral sucker posteriad or from ventral sucker posteriad. Dorsolateral and ventrolateral nerve chords present.

Oral sucker robust, aspinous, demarcated from body by posterior constriction. Ventral sucker present, aspinous. Pharynx present, enveloping anterior extremity of oesophagus. Oesophagus extending posteriad $<1 / 4$ of body length, ventral to anterior nerve commissure, lacking diverticula, plicate organ, or median oesophageal pouch, straight or slightly sinuous; oesophageal gland surrounding oesophagus from posterior margin of pharynx to oesophageal-intestinal junction, strongly basophilic, widest at level of caecal bifurcation. Intestine comprising non-fused caeca, inverse U-shaped, smooth (lacking diverticula or secondary rami), extending $1 / 2-3 / 4$ of body length directly posteriad, not extensively convoluted, bifurcating anterior to ventral sucker, terminating in posterior body extremity.

Testes comprising one anterior testis and one posterior testis, in posterior half of body, intercaecal, having deep lobes or slightly irregular margins. Vas deferens extending directly anteriad and ventral to cirrus sac before turning posteriad and expanding to form external seminal vesicle; external seminal vesicle posterior to ventral sucker, intercaecal, abutting anterodextral margin of cirrus sac, at level of common genital pore. Cirrus sac robust, pre-testicular, containing variously-sized secretory cells and large pars prostatica.

Ovary single, wider than long, triangular, lacking lobes, intercaecal, intertesticular. Oviduct emerging from dextral margin of ovary, directing posteriad; oviducal seminal receptacle comprising middle portion of oviduct between ovary and posterior testis. Laurer's canal intercaecal, intertesticular, post-ovarian, extending anteriad or posteriad from oviduct at level of vitelline reservoir, opening dorsally. Vitellarium follicular, occupying space from caecal bifurcation to caecal termination; vitelline reservoir intercaecal, intertesticular, ventral to ovary. Ootype a weakly glandular and thin-walled chamber, intercaecal, occupying space between anterior testis and ovary, posterior to cirrus sac.

Uterus intercaecal, intertesticular, straight (not coiled); uterine pouch absent. Egg single, occupying female reproductive tract proximal to metraterm. Metraterm massive, approximately $3-7 \times$ uterus length, longitudinal (extending anteriad in parallel with body margin), between ventral sucker and vitelline reservoir, sinistral to anterior testis, having an obviously muscular thick wall, apparently not storing egg(s). Common genital pore dorsal, sinistral, posterior to ventral sucker, predominantly anterior to genitalia, aspinous, lacking suckers.

Excretory vesicle globular, extensively lobed, extending posteriad from distal ends of caeca to posterior body end.
Excretory pore terminal. Manter's organ absent. In blood of Asiatic trionychids.

Differential diagnosis. Body 3-7× longer than wide, aspinous; ventrolateral tegumental papillae present. Ventral sucker present. Oesophagus $<1 / 4$ of body length, lacking diverticula, plicate organ, or median oesophageal pouch. Intestine comprising non-fused caeca. Testes comprising one anterior and one posterior testis. External seminal vesicle abutting anterodextral margin of cirrus sac and at level of common genital pore. Ovary intertesticular. Oviducal seminal receptacle comprising middle portion of oviduct between ovary and posterior testis. Laurer's canal intercaecal, intertesticular, post-ovarian. Vitelline reservoir intertesticular. Uterine pouch absent. Egg single, occupying female reproductive tract proximal to metraterm. Metraterm massive, approximately 3-7× uterus length, apparently not storing egg(s). Common genital pore dorsal, sinistral, predominantly anterior to genitalia.

Type species: Coeuritrema lyssimus Mehra, 1933 from heart ventricle of Indian flapshell turtle, Lissemys punctata (Bonnaterre) (Testudines: Trionychidae), in the Ganges River, India.

Remarks. Mehra (1933) proposed Coeuritrema for C. lyssimus and Hapalorhynchus odhnerensis (Mehra, 1933) Byrd, 1939. That diagnosis included "body wall with or without small papillae" (= ventrolateral tegumental papillae) such that $H$. odhnerensis, which lacks ventrolateral tegumental papillae, could be included. As emended herein, however, Coeuritrema includes papillate TBFs only. This genus has long been regarded as a junior subjective synonym of Hapalorhynchus (see Byrd 1939, Hughes et al. 1942, Brooks and Mayes 1976, Platt 1988, 2002, Bourgat 1990), which lacks ventrolateral tegumental papillae. Coeuritrema further differs from Hapalorhynchus by having an external seminal vesicle that abuts the anterodextral margin of the cirrus sac and a definitive metraterm that is approximately $3-7 \times$ the uterus length.

The ootype and uterus are both relatively short and together, rather than the metraterm, may store the egg in species of Coeuritrema. If a large egg, presumably one that is developed and ready for passage to the metraterm and/or for ejection, is present in the proximal portion of the female reproductive tract, the egg occupies the luminal spaces of both the ootype and uterus (Fig. 4). In that case, the ootype and uterus are difficult to differentiate (Fig. 4). Without an egg, the clear distinction between the ootype and the uterus is evident: the ootype comprises the laterally-expanded chamber immediately distal to the ovo-vitelline duct and the uterus is the narrow, short duct connecting it with the metraterm (Fig. 6). If our understanding is correct, it may be useful to consider the ootype and uterus functioning together as an 'egg chamber'. Noteworthy along these lines also is that we did not observe an egg in the metraterm of any specimen, indicating that perhaps the metraterm of Coeuritrema spp. does not retain the egg.

These observations match those of previous workers handling specimens of the type species and Coeuritrema rugatus. Mehra (1933) explicitly reported that he never ob- 
served more than a single egg ("ovum") in the female reproductive tract of C. lyssimus (type species) and that each egg resided "in the proximal portion of the metraterm," which, based on his illustrations (see plate 1, fig. 1 of Mehra 1933) could be interpreted as the putative egg chamber. Brooks and Sullivan (1981) likewise reported that only a single egg occurred in the uterus of ovigerous specimens. Perhaps this is related to the gestation time for the large egg.

The metraterm of other whole-mounted blood flukes does hold eggs (Bullard and Overstreet 2006, Bullard et al. 2006, Bullard and Jensen 2008); however, eggs in those species are smaller, much more numerous in the uterus and metraterm, and seemingly less developed. Perhaps this is because they must infect minute branchial vessels before transiting to the gill epithelium and hatching (Bullard and Overstreet 2008). The extent to which the metraterm serves as a storage organ for TBF eggs is indeterminate, but if particular genera have or lack such an 'egg chamber' (= ootype + uterus), it is likely a significant differential diagnostic feature relating genera. Resin-based ultramicrotomy of the wall of the proximal portion of the female reproductive tract could reveal cellular changes to the putative egg chamber wall in the presence/absence of the large egg.

Coeuritrema and Hapalorhynchus are similar morphologically by having a ventral sucker, non-fused caeca, two testes, a pre-testicular cirrus sac, an intertesticular ovary, and a common genital pore that opens dorsally and in the sinistral half of the body. Phylogenetic analysis of the D1-D3 domains of the nuclear large subunit ribosomal DNA (28S) suggests that Coeuritrema and Hapalorhynchus indeed share a recent, common ancestor (Fig. 7; see below). We concur with Platt (2002) in that several apapillate turtle blood fluke species originally assigned to Coeuritrema should be assigned to Hapalorhynchus: H. odhnerensis, Hapalorhynchus ocadiae (Takeuti, 1942), Hapalorhynchus oschmarini (Belous, 1963), Hapalorhynchus macrotesticularis (Rohde, Lee et Lim, 1968), Hapalorhynchus mica (Oshmarin, 1971), Hapalorhynchus sheilae (Mehrotra, 1973) and Hapalorhynchus sutlejensis (Mehrotra, 1973) (see Takeuti 1942, Rohde et al. 1968, Oshmarin 1971, Mehrotra 1973). This brings the total number of accepted species of Coeuritrema and Hapalorhynchus to 3 and 19 , respectively.

Yamaguti (1958) considered Coeuritrema a junior synonym of Tremarhynchus Thapar, 1933; however, the diagnosis of Coeuritrema was published in May 1933 and that of Tremarhynchus was published immediately thereafter in June. Hence, the former genus has taxonomic priority (Dwivedi 1967). Byrd (1939) and Platt (2002) considered Tremarhynchus (and Coeuritrema) a junior subjective synonym of Hapalorhynchus. No author has treated Tremarhynchus since 2002.

Tremarhynchinae Yamaguti, 1958 included Enterohaematotrema and Tremarhynchus (type genus) (see Yamaguti 1958). Dwivedi (1967) commented that the subfamily should be renamed Coeuritrematinae Dwivedi, 1967 (type genus Coeuritrema) to correct Yamaguti's (1958) name for the subfamily based on his erroneous acceptance of
Tremarhynchus as a senior synonym of Coeuritrema by taxonomic priority. This decision was probably based upon the International Code of Zoological Nomenclature's Article 61.1.2. (under "Statement of the Principle of Typification"), which states that "a nominal family-group taxon is the nominal genus on which its name is based." (International Commission on Zoological Nomenclature 2000). Hence, because post-1967 Coeuritrema was correctly understood to have taxonomic priority over Tremarhynchus, the subfamily name had to be changed.

Yamaguti (1971) accepted Coeuritrematinae Dwivedi, 1967 (as "1968") and included Coeuritrema, Enterohaematotrema, and Cardiotrema based on the presence of a ventral sucker, paired caeca not united posteriorly, two testes, a cirrus sac "more or less strongly developed" between the ventral sucker and anterior testis, an intertesticular ovary, a vitellarium as long as caeca, and a genital pore between the ventral sucker and testes. These genera plus Hapalorhynchus and Vasotrema include species infecting softshell turtles (Trionychidae) (Table 1). No molecular sequence data exist for any species of Enterohaematotrema or Cardiotrema, and Vasotrema does not group with the Coeuritrema + Hapalorhynchus clade (Fig. 7). Hence, presently, no support exists for monophyly of softshell TBFs, suggesting that TBFs have repeatedly, independently colonised softshell turtles.

Coeuritrema differs from Enterohaematotrema by having a dorsal, sinistral genital pore (rather than a ventral, medial genital pore), from Cardiotrema by having an intertesticular ovary (rather than a sinistral ovary) and a large ventral sucker $>1 / 2$ body width (rather than $<1 / 5$ body width for Cardiotrema), and from Vasotrema by having two testes and a dorsal genital pore (rather than one testis posterior to the ovary and a ventral genital pore). Mehra (1934) provided a key to TBF genera and differentiated Coeuritrema and Hapalorhynchus by the presence or absence of a cirrus sac and cirrus. These features have been the focus of intense study because of their stated value as differential features. Our observations herein confirmed the presence of an obvious cirrus sac among the accepted species of Coeuritrema, but this feature may or may not be of generic importance in other TBF genera. For example, within the closely-related Hapalorhynchus the presence or absence of the cirrus sac and cirrus have been accepted as generic features by some (Mehra 1939, 1940, Yamaguti 1958,1971 ) and rejected by others (Byrd 1939, Brooks and Mayes 1976, Platt 1988, 2002, Bourgat 1990).

Brooks and Mayes (1976) provided supplemental observations of the male genitalia of Hapalorhynchus stunkardi Byrd, 1939 and emended Hapalorhynchus to include "well-developed or poorly-developed cirrus." Platt (1988) observed, in histological sections, prostate cells not bound by a limiting membrane but scattered in the surrounding parenchyma in Hapalorhynchus gracilis Stunkard, 1922, H. foliorchis and H. stunkardi. He also observed that the prostatic complex of Hapalorhynchus albertoi Lamothe-Argumedo, 1978 and Hapalorhynchus brooksi Platt, 1988 was membrane-bound (= cirrus sac) and that the distal portion of the ejaculatory duct (= cirrus) was eversi- 
Table 1. Blood flukes infecting softshell turtles (Testudines: Trionychidae)

\begin{tabular}{|c|c|c|c|c|c|}
\hline Turtle & Fluke & Site in host & Locality & Museum nos. & Reference \\
\hline $\begin{array}{l}\text { Amyda cartilaginea } \\
\text { (Boddaert), Asiatic } \\
\text { softshell }\end{array}$ & $\begin{array}{l}\text { Coeuritrema rugatus } \\
\text { (Brooks et Sullivan, 1981) } \\
\text { comb. } \mathrm{n} .\end{array}$ & $\begin{array}{l}\text { mesenteric blood } \\
\text { vessels }\end{array}$ & $\begin{array}{l}\text { Sungei Jempol, Ulu Jempol, State of Negeri } \\
\text { Sembilan, West Malaysia }\end{array}$ & HWML 21339 & $\begin{array}{l}\text { Brooks and Sullivan } \\
\text { (1981) }\end{array}$ \\
\hline \multirow[t]{4}{*}{$\begin{array}{l}\text { Apalone ferox (Schneider), } \\
\text { Florida softshell }\end{array}$} & $\begin{array}{l}\text { Vasotrema amydae Stunk- } \\
\text { ard, } 1926 \text { (type species) }\end{array}$ & blood & $\begin{array}{l}\text { probably a Gulf of Mexico river drainage, } \\
\text { Florida, USA }\end{array}$ & AMNH 791 & Stunkard (1926) \\
\hline & & blood & $\begin{array}{l}\text { probably a Gulf of Mexico river drainage, } \\
\text { Florida, USA }\end{array}$ & AMNH 791 & Stunkard (1928) \\
\hline & $\begin{array}{l}\text { Vasotrema attenuatum } \\
\text { Stunkard, } 1928\end{array}$ & blood & $\begin{array}{l}\text { probably a Gulf of Mexico river drainage, } \\
\text { Florida, USA }\end{array}$ & AMNH 806, 807 & Stunkard (1928) \\
\hline & Vasotrema sp. & not specified & $\begin{array}{l}\text { Apalachicola River, Franklin County, } \\
\text { Florida, USA }\end{array}$ & not specified & Loftin (1960) \\
\hline \multirow{3}{*}{$\begin{array}{l}\text { Apalone mutica (LeSueur), } \\
\text { smooth softshell }\end{array}$} & Vasotrema attenuatum & blood & Missouri River Drainage, Nebraska, USA & not specified & Brooks and Mayes (1975) \\
\hline & $\begin{array}{l}\text { Vasotrema brevitestis } \\
\text { Brooks et Mayes, } 1975\end{array}$ & blood & $\begin{array}{l}\text { Missouri River, } 2.4 \mathrm{~km} \text { south of Blair, } \\
\text { Nebraska, USA }\end{array}$ & $\begin{array}{l}\text { USNM } \\
73817-73819 \\
\text { HWML } 20077\end{array}$ & Brooks and Mayes (1975) \\
\hline & Vasotrema robustum & blood & Missouri River Drainage, Nebraska, USA & not specified & Brooks and Mayes (1975) \\
\hline \multirow[t]{12}{*}{$\begin{array}{l}\text { Apalone spinifera } \\
\text { (LeSueur), spiny softshell }\end{array}$} & $\begin{array}{l}\text { Vasotrema amydae } \\
\text { (type species) }\end{array}$ & blood & Ohio River Drainage, Indiana, USA & AMNH 791 & Stunkard (1926) \\
\hline & & blood & Ohio River Drainage, Indiana, USA & AMNH 791 & Stunkard (1928) \\
\hline & Vasotrema attenuatum & blood & Ohio River Drainage, Indiana, USA & AMNH 806, 807 & Stunkard (1928) \\
\hline & & $\begin{array}{l}\text { mesenteric blood } \\
\text { vessels }\end{array}$ & Reelfoot Lake, Tennessee, USA & HWML9227 & $\begin{array}{l}\text { Byrd (1939) (see also } \\
\text { Platt and Snyder 2007) }\end{array}$ \\
\hline & & not specified & Missouri River Drainage, Nebraska, USA & not specified & Brooks and Mayes (1975) \\
\hline & Vasotrema brevitestis & not specified & $\begin{array}{l}\text { Atkinson Lake, Atkinson Recreation Area, } \\
0.8 \mathrm{~km} \text { west of Atkinson, Nebraska, USA }\end{array}$ & $\begin{array}{l}\text { USNM } \\
\text { 73817-73819; } \\
\text { HWML 20076 }\end{array}$ & Brooks and Mayes (1975) \\
\hline & $\begin{array}{l}\text { Vasotrema longitestis } \\
\text { Byrd, } 1939\end{array}$ & arterial circulation & Reelfoot Lake, Tennessee, USA & $\begin{array}{l}\text { USNM 9229, } \\
80656-8 ; \text { HWML } \\
31121-8\end{array}$ & $\begin{array}{l}\text { Byrd (1939) (see Platt and } \\
\text { Prestwood 1990) }\end{array}$ \\
\hline & Vasotrema robustum & blood & Ohio River Drainage, Indiana, USA & AMNH 808, 809 & Stunkard (1928) \\
\hline & & arterial system & Reelfoot Lake, Tennessee, USA & $\begin{array}{l}\text { USNM 80657-2; } \\
\text { HWML 31122-2 }\end{array}$ & $\begin{array}{l}\text { Byrd (1939) (see Platt and } \\
\text { Prestwood 1990) }\end{array}$ \\
\hline & & $\begin{array}{l}\text { heart, large blood } \\
\text { vessels }\end{array}$ & $\begin{array}{l}\text { Huron River, Washtenaw County, Michigan, } \\
\text { USA }\end{array}$ & USNM 37306 & Wall (1951) \\
\hline & & not specified & Missouri River Drainage, Nebraska, USA & HWML 20075 & Brooks and Mayes (1975) \\
\hline & & not specified & Nishnabotna River, Floyd County, Iowa, USA & HWML 45795 & Snyder (2004) \\
\hline $\begin{array}{l}\text { Dogania subplana } \\
\text { (Saint-Hilaire), } \\
\text { Malayan softshell turtle }\end{array}$ & $\begin{array}{l}\text { Hapalorhynchus } \\
\text { macrotesticularis (Rohde, } \\
\text { Lee et Lim, 1968) }\end{array}$ & $\begin{array}{l}\text { heart, arteries near } \\
\text { heart }\end{array}$ & $\begin{array}{l}\text { unknown drainage, Kuala Lumpur } \\
\text { (purchased from Chinese merchant), } \\
\text { Malaysia }\end{array}$ & $\begin{array}{l}\text { Helm. Coll. No. } \\
\text { R. } 769 \text {, Zoology } \\
\text { Dept., University } \\
\text { of Malaysia, Kuala } \\
\text { Lumpur }\end{array}$ & Rohde et al. (1968) \\
\hline \multirow[t]{10}{*}{$\begin{array}{l}\text { Lissemys punctata (Bonna- } \\
\text { terre), Indian flapshell }\end{array}$} & $\begin{array}{l}\text { Cardiotrema roparensis } \\
\text { (Mehrotra, 1973) }\end{array}$ & not specified & Sutlej River, Ropar, Punjab State, India & not specified & Mehrotra (1973) \\
\hline & & $\begin{array}{l}\text { body wash, hepatic } \\
\text { blood vessels }\end{array}$ & $\begin{array}{l}\text { Sutlej River, Ropar, Punjab State; Yamuna } \\
\text { River, Karnal, Haryana State, India }\end{array}$ & not specified & Tandon and Gupta (1985) \\
\hline & $\begin{array}{l}\text { Enterohaematotrema } \\
\text { palaeorticum Mehra, } 1940 \\
\text { (type species) }\end{array}$ & $\begin{array}{l}\text { small intestine } \\
\text { (probably mesenter- } \\
\text { ic blood vessels) }\end{array}$ & $\begin{array}{l}\text { Ganges River, Allahabad, Uttar Pradesh } \\
\text { - State, India }\end{array}$ & not specified & Mehra (1940) \\
\hline & $\begin{array}{l}\text { Coeuritrema lyssimus } \\
\text { Mehra, } 1940 \text { (type species) }\end{array}$ & $\begin{array}{l}\text { ventricle of heart } \\
\text { (adult) }\end{array}$ & $\begin{array}{l}\text { Ganges River, Allahabad, Uttar Pradesh } \\
\text { State, India }\end{array}$ & not specified & Mehra (1933) \\
\hline & & heart (adult) & $\begin{array}{l}\text { Ganges River Drainage, Rudrapur, } \\
\text { Uttarakhand State, India }\end{array}$ & not specified & Tandon and Gupta (1982) \\
\hline & $\begin{array}{l}\text { Hapalorhynchus } \\
\text { odhnerensis (Mehra, 1940) }\end{array}$ & ventricle of heart & $\begin{array}{l}\text { Ganges River, Allahabad, Uttar Pradesh } \\
\text { State, India }\end{array}$ & not specified & Mehra (1933) \\
\hline & $\begin{array}{l}\text { Hapalorhynchus sheilae } \\
\text { (Mehrotra, 1973) }\end{array}$ & not specified & $\begin{array}{l}\text { Patiala River (Ghaggar River Drainage), } \\
\text { Patiala, Punjab State; Sutlej River, Ropar, } \\
\text { Punjab State; Ghaggar River, Sangrur, } \\
\text { Punjab State; Ganges River Drainage, } \\
\text { Rudrapur, Uttarakhand State, India }\end{array}$ & not specified & Mehrotra (1973) \\
\hline & & heart, blood, liver & $\begin{array}{l}\text { Ganges River Drainage, Rudrapur, Uttara- } \\
\text { khand State; Patiala River (Ghaggar River } \\
\text { Drainage), Patiala, Punjab State; Ghaggar } \\
\text { River, Sangrur, Punjab State, India }\end{array}$ & not specified & Tandon and Gupta (1982) \\
\hline & $\begin{array}{l}\text { Hapalorhynchus sutlejensis } \\
\text { (Mehrotra, 1973) }\end{array}$ & not specified & $\begin{array}{l}\text { Sutlej River, Ropar, Punjab State; Ghaggar } \\
\text { River, Sangrur, Punjab State, India }\end{array}$ & not specified & Mehrotra (1973) \\
\hline & & ventricle of heart & $\begin{array}{l}\text { Gomti River, Lucknow, Uttar Pradesh State; } \\
\text { Ghaggar River, Sangrur, Punjab State, India }\end{array}$ & not specified & Tandon and Gupta (1982) \\
\hline $\begin{array}{l}\text { Nilssonia gangetica (Cuvi- } \\
\text { er), Indian softshell }\end{array}$ & $\begin{array}{l}\text { Hapalorhynchus indicus } \\
\text { (Thapar, 1933) }\end{array}$ & $\begin{array}{l}\text { heart, large blood } \\
\text { vessels }\end{array}$ & $\begin{array}{l}\text { Gomti River, Lucknow, Uttar Pradesh } \\
\text { State, India }\end{array}$ & not specified & Thapar (1933) \\
\hline $\begin{array}{l}\text { Nilssonia hurum (Gray), } \\
\text { Indian peacock softshell }\end{array}$ & $\begin{array}{l}\text { Enterohaematotrema } \\
\text { palaeorticum (type species) }\end{array}$ & $\begin{array}{l}\text { intestine (probably } \\
\text { mesenteric blood } \\
\text { vessels) }\end{array}$ & $\begin{array}{l}\text { pond in Kotah, Raipur, Madhya Pradesh } \\
\text { State, India }\end{array}$ & not specified & Shrivastava (1959) \\
\hline \multirow[t]{3}{*}{$\begin{array}{l}\text { Pelodiscus sinensis (Wieg- } \\
\text { mann), Chinese softshell }\end{array}$} & Coeuritrema platti sp. n. & $\begin{array}{l}\text { heart, mesentery, } \\
\text { lung }\end{array}$ & Da Rang River, Phuyen Province, Vietnam & $\begin{array}{l}\text { USNM 1411790, } \\
1411791\end{array}$ & Present study \\
\hline & $\begin{array}{l}\text { Hapalorhynchus mica } \\
\text { (Oshmarin, 1971) }\end{array}$ & $\begin{array}{l}\text { hepatic blood } \\
\text { vessels }\end{array}$ & Duong River, Hai Phong, Vietnam & not specified & Oshmarin (1971) \\
\hline & $\begin{array}{l}\text { Hapalorhynchus } \\
\text { oschmarini (Belous, 1963) }\end{array}$ & $\begin{array}{l}\text { hepatic blood } \\
\text { vessels }\end{array}$ & Lake Khanka, Russia & not specified & Belous (1963) \\
\hline
\end{tabular}


ble (Lamothe-Argumedo 1978, Platt 1988). As a result, he emended the diagnosis as "cirrus sac present or absent." As additional species of Coeuritrema are documented, special care should be taken to assess the cirrus sac and cirrus.

Coeuritrema rugatus (Brooks et Sullivan, 1981) comb. n. Figs. 1, 2

\section{ZooBank number for species:}

urn:1sid:zoobank.org:act:36DE9E3C-2809-476B-978A-67B2CC3B122F

Redescription (based on light microscopy of eight whole-mounted adult specimens, including paratypes): Body $1250-1540(1423 ; 7)$ long or $5.3-7.2 \times(6.5 ; 7)$ longer than wide, $150-200(177 ; 8)$ wide or $12-13 \%(12 \%$; 7) of body length at level of caecal bifurcation, 200-265 $(219 ; 8)$ wide or $14-19 \%(15 \% ; 7)$ of body length at ovary (typically maximum width of specimen), 150-205 (174; 8) wide or $11-15 \%(12 \% ; 7)$ of body length at caecal termination; forebody (middle of ventral sucker to anterior end) $340-470(439 ; 7)$ long or $24-34 \%(31 \% ; 7)$ of body length, lacking ventrolateral tegumental papillae; hindbody (middle of ventral sucker to posterior end) 840-1090 (968; 8) long or $66-76 \%(69 \% ; 7)$ of body length, having ventrolateral tegumental papillae (Fig. 1). Ventrolateral tegumental papillae 17-20 $(19 ; 8)$ along dextral body margin, 18-20 $(19 ; 8)$ along sinistral body margin, $35-39(38 ; 8)$ total (Fig. 1); anterior papillae 8-35 (20; 8 [3 papillae measured per specimen]) long, with base $15-38$ (24; 8) wide, $0.6-2.5 \times(1.3 ; 8)$ wider than long; middle papillae $25-43$ $(32 ; 8)$ long, with base $35-53(43 ; 8)$ wide, $1.0-1.8 \times(1.3$; $8)$ wider than long; posterior papillae $8-38(23 ; 8)$ long, base $13-45(29 ; 8)$ wide, $0.7-2.9 \times(1.3 ; 8)$ wider than long.

Oral sucker $60-75(67 ; 7)$ long or $4-5 \%(5 \% ; 7)$ of body length, $78-100(93 ; 7)$ wide or $38-50 \%(43 \%$; 7) of maximum body width (Fig. 1). Ventral sucker 103-125 (114; 8) long or $7-9 \%(8 \% ; 7)$ of body length, $103-133(120 ; 8)$ wide or $57-75 \%(68 \% ; 8)$ of body width at caecal bifurcation, $1.3-1.5 \times(1.3 ; 7)$ wider than oral sucker (Fig. 1). Nerve commissure $193-225(202 ; 7)$ or $13-16 \%(14 \% ; 7)$ of body length from anterior body end.

Pharynx $53-68(59 ; 7)$ long or $20-25 \%(23 \% ; 7)$ of oesophagus length, $60-83(77 ; 7)$ wide or $0.8-1.5 \times(1.1$; 7) maximum oesophagus width (Fig. 1). Oesophagus $225-295(261 ; 7)$ long or $17-21 \%(18 \%$; 7$)$ of body length, $15-25(19 ; 7)$ wide posterior to pharynx, with wall $10-18$ $(14 ; 7)$ thick, 53-95 $(73 ; 8)$ maximum width or $29-53 \%$ $(41 \% ; 8)$ of body width at caecal bifurcation, with wall $10-50(24 ; 8)$ thick (Fig. 1), narrowing to $13-25(20 ; 8)$ width or $7-14 \%(11 \% ; 8)$ of body width at caecal bifurcation, with wall $10-20(15 ; 8)$ thick; oesophageal gland $205-245(223 ; 8)$ long or $14-17 \%(16 \% ; 7)$ of body length and $81-93 \%(86 \% ; 7)$ of oesophagus length, 100-135 $(121 ; 8)$ wide or $56-84 \%(70 \% ; 8)$ of body width at caecal bifurcation.

Intestine bifurcating $262-330(285 ; 7)$ or $17-23 \%$ $(20 \% ; 7)$ of body length from anterior end; sinistral caecum $850-1050(962 ; 8)$ long or $67-72 \%(69 \% ; 7)$ of body length, $73-100(87 ; 7)$ wide at bifurcation or $46-54 \%$
$(49 \% ; 7)$ of body width, 20-30 $(26 ; 8)$ wide at ovary or $9-15 \%(12 \% ; 8)$ of body width, $23-45(31 ; 8)$ wide at termination or $13-24 \%(18 \% ; 8)$ of body width; dextral caecum $820-1100(953 ; 8)$ long or $65-73 \%(68 \% ; 7)$ of body length, $65-90(77 \% ; 8)$ wide at bifurcation or $38-50 \%$ $(43 \% ; 8)$ of body width, $15-30(24 ; 8)$ wide at ovary or $7-13 \%(11 \% ; 8)$ of body width, $25-45(32 ; 8)$ wide at termination or $15-24 \%(18 \% ; 8)$ of body width; caecal termination $115-145(130 ; 8)$ or $8-10 \%(9 \% ; 7)$ from posterior end.

Anterior testis lobed, intensely follicular marginally, $118-188(143 ; 8)$ long or $9-13 \%(10 \% ; 7)$ of body length, $70-115(87 ; 8)$ wide or $35-44 \%(40 \% ; 8)$ of body width at gonads; intertesticular space $63-90(83 ; 8)$ or $4-7 \%(6 \%$; 7) of body length (Figs. 1, 2). Posterior testis as anterior testis, $138-208(167 ; 8)$ long or $10-15 \%(12 \% ; 7)$ of body length, $70-135(91 ; 8)$ wide or $35-51 \%(41 \% ; 7)$ of body width at gonads; post-testicular space $340-430(391 ; 8)$ or $26-30 \%(28 \% ; 7)$ of body length from posterior end. Anterior trunk of vasa efferentia emanating from ventral surface of anterior testis, extending anteriad 20-28 $(22 ; 8)$ or $<1 \%$ (7) of body length from testis, 5 (8) wide or $2-3 \%(2 \% ; 8)$ of body width (Fig. 2); posterior trunk of vasa efferentia emanating from ventral surface of posterior testis, extending anteriad $200-273(234 ; 8)$ or $16-19 \%(17 ; 7)$ of body length, $3-5(4 ; 8)$ wide or $1-3 \%(2 \% ; 8)$ of body width, coalescing with anterior trunk of vasa efferentia ventral to anterior portion of anterior testis (Fig. 2).

Vas deferens $88-153(111 ; 8)$ long or $6-10 \%(8 \% ; 7)$ of body length, $5-8(5 ; 8)$ in maximum width, extending anteroventrally. External seminal vesicle 40-80 $(60 ; 8)$ long or $3-6 \%(4 \% ; 7)$ of body length, $25-115(46 ; 8)$ wide, $0.7-2.0 \times(1.5 ; 8)$ longer than wide, narrowing posteriorly and proximal to internal seminal vesicle (Figs. 1, 2); internal seminal vesicle $75-88(80 ; 8)$ long or $1.0-2.1 \times(1.4$; $8)$ external seminal vesicle length, $25-35(31 ; 8)$ wide or $2.2-3.1 \times(2.6 ; 8)$ longer than wide or $22-132 \%(81 \% ; 8)$ of external seminal vesicle width (Figs. 1, 2). Pars prostatica surrounding distal portion of internal seminal vesicle, $48-58(52 ; 8)$ long, $30-35(32 ; 8)$ wide or $1.5-1.9 \times(1.6$; 8) longer than wide (Fig. 2). Cirrus 5-10 (8; 8) long, 3-5 $(4 ; 8)$ wide; cirrus sac enveloping most of internal seminal vesicle and entirety of pars prostatica, 88-160 $(119 ; 8)$ long or $6-11 \%(8 \% ; 7)$ of body length, $55-95(67 ; 8)$ wide or $24-44 \%(31 \% ; 8)$ of maximum body width, containing large putative secretory cells (Figs. 1,2).

Ovary triangular, with broadest portion in sinistral half of body, $260-355(310 ; 8)$ or $21-25 \%(22 \%$; 7$)$ of body length from middle of ventral sucker buttressing caeca, $58-100(77 ; 8)$ long or $5-6 \%(6 \% ; 7)$ of body length, $93-140(112 ; 8)$ wide or $44-58 \%(51 \% ; 7)$ of body width, $1.3-1.8 \times(1.5 ; 8)$ wider than long; post-ovarian space $495-640(581 ; 8)$ or $40-45 \%(41 \% ; 7)$ of body length (Fig. 1); oocytes largest and most basophobic near oviduct (Fig. 2). Oviduct extending posteriad, turning dorsally, proceeding counterclockwise for $48-65(54 ; 8)$ or $3-5 \%$ $(4 \% ; 7)$ of body length, $5-10(8 ; 8)$ in maximum width, expanding laterally to form oviducal seminal receptacle; oviducal seminal receptacle extending sinistrad for 53-80 
1

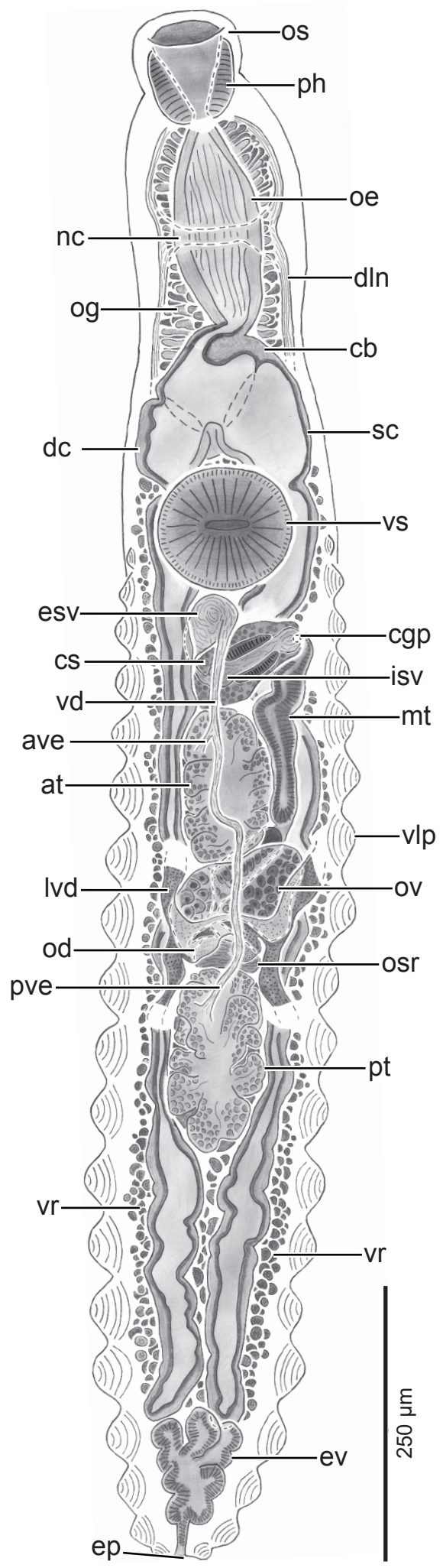

2

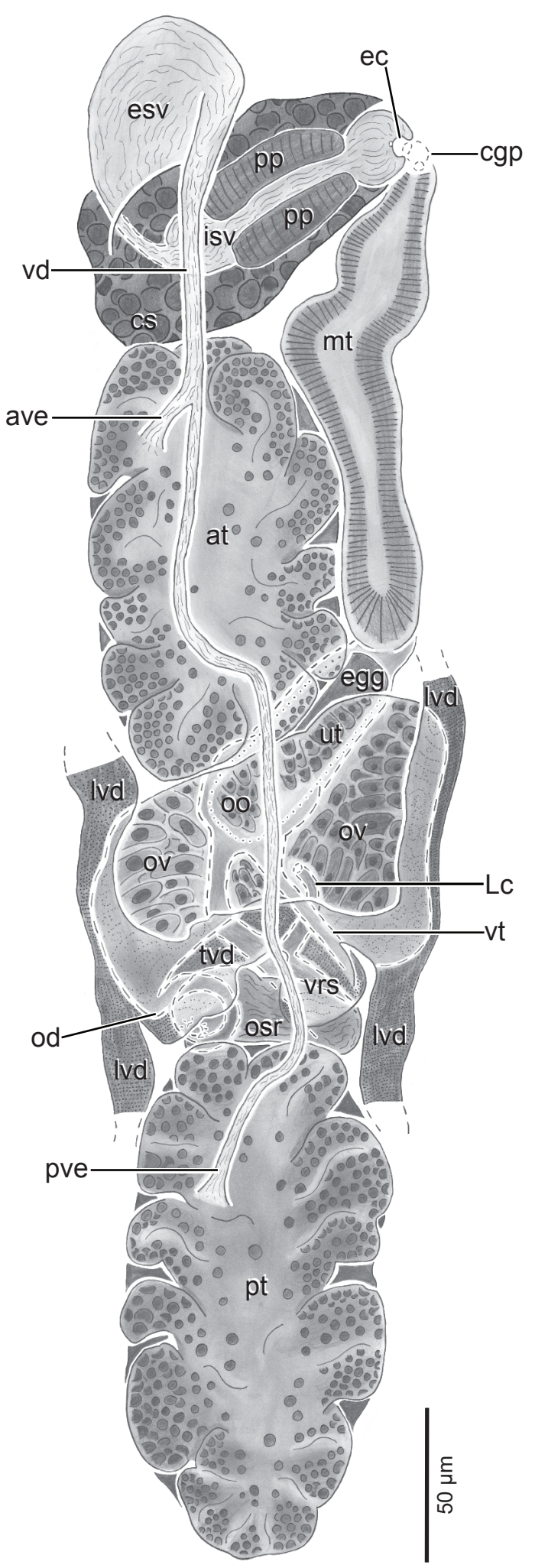

Figs. 1-2. Coeuritrema rugatus (Brooks et Sullivan, 1981) comb. n. (Digenea: Schistosomatoidea) from the mesenteric vessels of Amyda cartilaginea (Boddaert) (Testudines: Trionychidae) from Malaysia, paratype (HWML Coll. No. 21339). Fig. 1. Total view, ventrally. Fig. 2. Genitalia, ventral view. Abbreviations: at - anterior testis; ave - anterior trunk of vasa efferentia; cb - caecal bifurcation; cgp - common genital pore; cs - cirrus sac; dc - dextral caecum; dln - dorsolateral nerve chord; ec - eversible cirrus; egg - egg in utero; ep - excretory pore; esv - external seminal vesicle; ev - excretory vesicle; isv - internal seminal vesicle; Lc - Laurer's canal; lvd - lateral vitelline collecting duct; $\mathrm{mt}$ - metraterm; nc - nerve commissure; od - oviduct; oe - oesophagus; og - oesophageal gland; oo - ootype; os - oral sucker; osr - oviducal seminal receptacle; ov - ovary; ph - pharynx; pp - pars prostatica; pt - posterior testis; pve - posterior trunk of vasa efferentia; sc - sinistral caecum; tvd - transverse vitelline duct; ut - uterus; vd - vas deferens; vlp - ventrolateral tegumental papillae; vr - vitellarium; vrs - vitelline reservoir; vs - ventral sucker; vt - vitelline duct. 
$(63 ; 8)$ or $58-112 \%(84 \% ; 8)$ of ovary width, $25-33(28 ; 8)$ in maximum width at origin or $9-16 \%(13 \% ; 8)$ of maximum body width, narrowing before turning dorsal, extending anterodextrad $58-95(82 ; 8)$ or $5-7 \%(6 \% ; 7)$ of body length, $10-13(12 ; 8)$ in maximum width or $5-7 \%(5 \% ; 8)$ of body width (Fig. 2). Laurer's canal a narrow duct extending $23-43(31 ; 8)$ anterosinistrad from middle portion of oviduct, $8-13(11 ; 8)$ wide, opening dorsally at level of middle portion of ovary (Fig. 2).

Vitellarium comprising a series of interconnected spheroid masses of follicles, distributing from level of caecal bifurcation to distal ends of caeca, lateral collecting ducts coalescing at level of posterior margin of ovary to form transverse vitelline duct; transverse vitelline duct ventral to ovary, $315-415(369 ; 8)$ or $25-29 \%(27 \%$; 7$)$ of body length from middle of ventral sucker (Figs. 1, 2); vitelline reservoir sac-like, ventral to oviducal seminal receptacle; vitelline duct extending anterodextrad and dorsal 55-85 $(64 ; 8)$ or $4-6 \%(5 \% ; 7)$ of body length before connecting with oviduct at ootype (Fig. 2). Ootype difficult to discern in gravid specimens, 30-43 $(36 ; 8)$ long, 28-43 $(37 ; 8)$ wide, dorsal to anterior margin of ovary (Fig. 2).

Uterus comprising proximal portion and metraterm (Fig. 2), with a single egg in seven of eight specimens; proximal portion of uterus extending anterosinsitrad from ootype, $28-50(39 ; 8)$ long or $2-4 \%(3 \% ; 7)$ of body length, $18-30(27 ; 8)$ wide or $9-15 \%(12 \% ; 8)$ of maximum body width; metraterm extending anterosinistrad, $148-218(175 ; 8)$ long or $11-15 \%(12 \% ; 7)$ of body length, maximum width of $25-45(34 ; 8)$ or $12-21 \%(16 \% ; 8)$ of maximum body width, $3.2-6.6 \times(4.7 ; 8)$ proximal uterus length. Uterine egg ovoid, 63-78 $(72 ; 7)$ long or 5\% (6) of body length, $23-33(27 ; 7)$ wide or $9-16 \%(12 \% ; 7)$ of maximum body width, $1.9-3.3 \times(2.7 ; 7)$ longer than wide (Fig. 2). Common genital pore $95-150(123 ; 8)$ or $8-10 \%$ $(9 \% ; 7)$ of body length posterior to middle of ventral sucker (Fig. 1).

Excretory vesicle $130-165(144 ; 8)$ long or $10-12 \%$ $(10 \% ; 7)$ of body length, $78-100(87 ; 8)$ wide or $45-55 \%$ $(50 \% ; 8)$ of body width at caecal termination; wall $8-13$ $(10 ; 8)$ thick (Fig. 1).

Type and only known host: Asiatic softshell turtle, Amyda cartilaginea (Boddaert) (Testudines: Trionychidae).

Ty p e l o c a lity: Sungei Jempol, Ulu Jempol, State of Negeri Sembilan, Malaysia.

Site in host: Mesenteric blood vessels.

Specimens examined: Hapalorhynchus rugatus - Harold W. Manter Laboratory (HWML) of Parasitology Coll. No. 21339, paratypes, eight slides comprising eight whole-mounted specimens, seven of the eight slides labeled as Hapalorhynchus rugosus, ex Amyda cartilaginea (as Trionyx cartilageneus) from Sungei Jempol, Ulu Jempol, State of Negeri Sembilan, Malaysia (Brooks and Sullivan 1981).

Remarks. Our interpretation of some features associated with type materials (see above) of C. rugatus contradicted those of Brooks and Sullivan (1981), who did not detail the vasa efferentia, vas deferens, ootype or Laurer's canal. They reported that $C$. rugatus lacked a pharynx; however, the paratypes have a large pharynx surrounding the anterior extremity of the oesophagus immediately posterior to the mouth (Fig. 1). Coeuritrema and Baracktrema Roberts, Platt et Bullard, 2016 are the only accepted TBF genera described as having a pharynx, and the pharynx is immediately posterior to the mouth and muscular oral sucker. Several species of Hapalorhynchus, Spirorchis MacCallum, 1918 and Vasotrema also have this configuration and will be treated in later taxonomic works (J.R.R., S.A.B. - unpubl. data).

Regarding the oviduct, Brooks and Sullivan (1981) illustrated it as originating from the posteromedial aspect of the ovary and turning anterodorsally, but we confirmed that it originates from the dextral side of the ovary. Regarding the oviducal seminal receptacle, they illustrated a sac-like structure branching from the oviduct and filled with sperm proximal to the uterus (fig. 3 in Books and Sullivan 1981, p. 1336). No paratype had such a structure. They stated that the vitelline reservoir was immediately postovarian and ventral to the ovary, but we observed that the reservoir coalesces sinistro-dorsal to the ovary (Fig. 2).

Coeuritrema platti Roberts et Bullard sp. n.

Figs. 3-7

ZooBank number for species:

urn:1sid:zoobank.org:act:539BDF18-65AD-40E6-B0F9-AB82923967B1

Description (based on light microscopy of seven whole-mounted adult specimens): Body 1035-1410 $(1218 ; 6)$ long or $5.5-6.9 \times(6.0 ; 6)$ longer than wide, $110-170(147 ; 6)$ wide or $11-15 \%(12 \% ; 6)$ of body length at level of caecal bifurcation, $150-225(203 ; 6)$ wide or $14-18 \%(17 \% ; 6)$ of body length at ovary (typically maximum width of specimen), $120-180(165 ; 6)$ wide or $12-16 \%(14 \% ; 6)$ of body length at caecal termination; forebody $390-519(444 ; 6)$ long or $32-39 \%(36 \% ; 6)$ of body length, hindbody $660-930(783 ; 6)$ long or $61-68 \%$ $(64 \% ; 6)$ of body length, having ventrolateral tegumental papillae (Figs. 3, 5). Ventrolateral tegumental papillae extending posteriad from middle of ventral sucker to posterior end 18-19 (18; 5) along dextral body margin, 17-20 (18; 5) along sinistral body margin, 35-39 $(36 ; 5)$ total (Figs. $3,5)$; anterior papillae $8-25(17 ; 3)$ long, with base $10-33$ $(21 ; 5)$ wide, $0.7-2.5 \times(1.2 ; 3)$ wider than long; middle papillae $10-25(17 ; 3)$ long, with base $18-35(26 ; 5)$ wide, $1.0-2.8 \times(1.7 ; 3)$ wider than long; posterior papillae 5-25 $(14 ; 3)$ long, with base $8-28(17 ; 5)$ wide, $0.6-2.0 \times(1.3$; 3) wider than long.

Oral sucker $45-70(58 ; 4)$ long or $4-6 \%(5 \% ; 4)$ of body length, $50-100(80 ; 6)$ wide or $33-45 \%(39 \%$; 6) of maximum body width (Figs. 3, 5). Ventral sucker 75-138 (115; 6) long or $7-13 \%(10 \% ; 6)$ of body length, 65-138 (114; $5)$ wide or $59-95 \%(77 \% ; 5)$ of body width at caecal bifurcation, $1.3-1.6 \times(1.4 ; 5)$ wider than oral sucker (Figs. 3, 5). Nerve commissure $150-238(188 ; 6)$ or $13-17 \%(15 \% ; 6)$ of body length from anterior body end.

Pharynx $45-63(58 ; 6)$ long or $20-25 \%(23 \% ; 6)$ of oesophagus length, $45-83(65 ; 6)$ wide or $1.6-2.2 \times(1.7$; 6) maximum oesophagus width (Figs. 3, 5). Oesophagus 
3

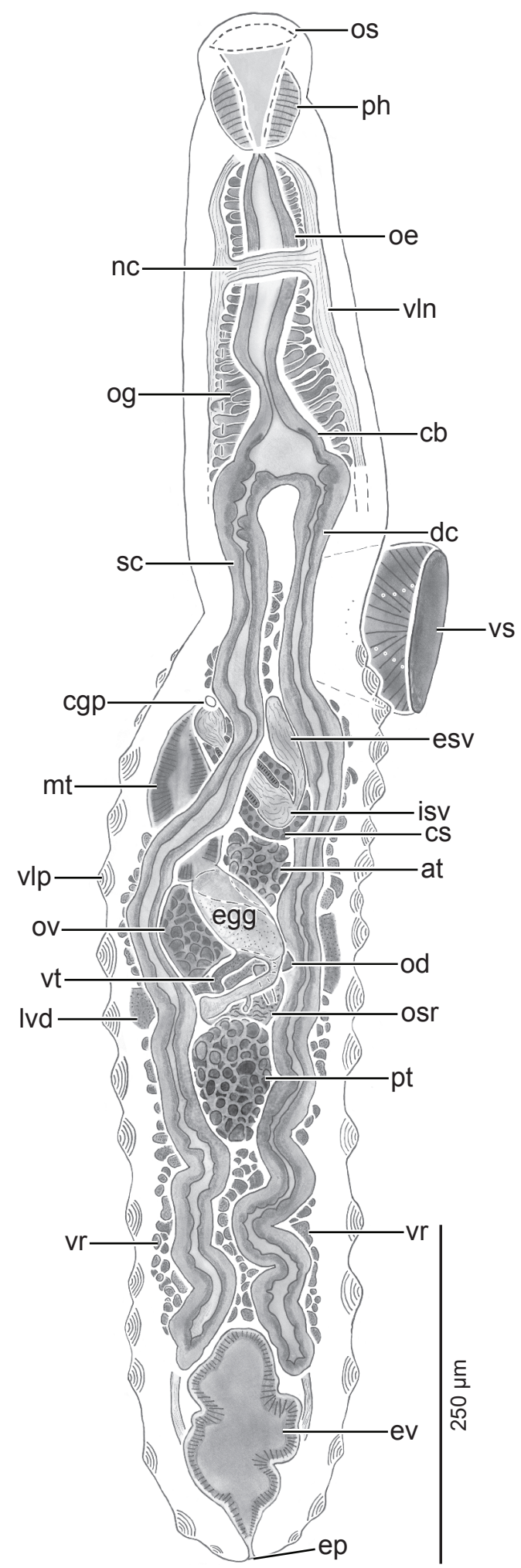

4

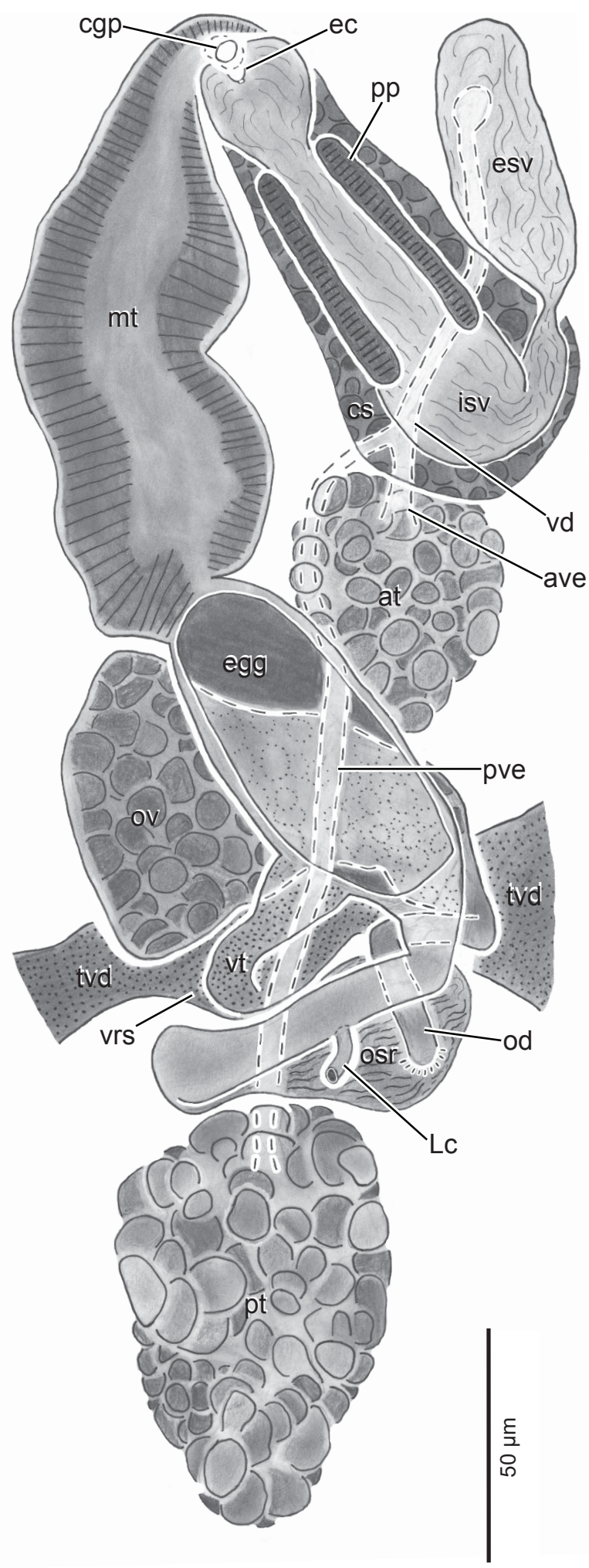

Figs. 3, 4. Coeuritrema platti sp. n. (Digenea: Schistosomatoidea) from viscera of Pelodiscus sinensis (Wiegmann) (Testudines: Trionychidae) from Vietnam, holotype (USNM Coll. No. 1411790). Fig. 3. Total view, dorsally. Fig. 4. Genitalia, dorsal view. Abbreviations: at - anterior testis; ave - anterior trunk of vasa efferentia; cb - caecal bifurcation; cgp - common genital pore; cs - cirrus sac; $\mathrm{dc}$ - dextral caecum; ec - eversible cirrus; egg - egg in utero; ep - excretory pore; esv - external seminal vesicle; ev - excretory vesicle; isv - internal seminal vesicle; Lc - Laurer's canal; lvd - lateral vitelline collecting duct; $\mathrm{mt}$ - metraterm; nc - nerve commissure; od - oviduct; oe - oesophagus; og - oesophageal gland; os - oral sucker; osr - oviducal seminal receptacle; ov - ovary; ph - pharynx; $\mathrm{pp}$ - pars prostatica; $\mathrm{pt}$ - posterior testis; pve - posterior trunk of vasa efferentia; sc - sinistral caecum; tvd - transverse vitelline duct; vd - vas deferens; vln - ventrolateral nerve chord; vlp - ventrolateral tegumental papillae; vr - vitellarium; vrs - vitelline reservoir; vs - ventral sucker; vt - vitelline duct. 
5

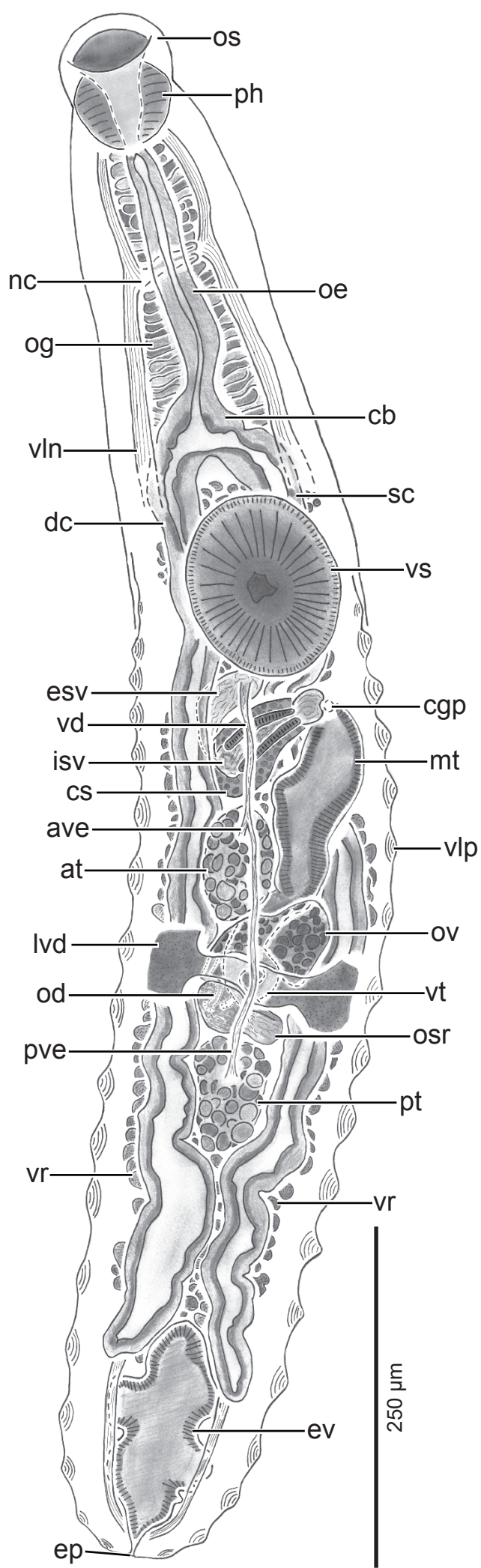

6

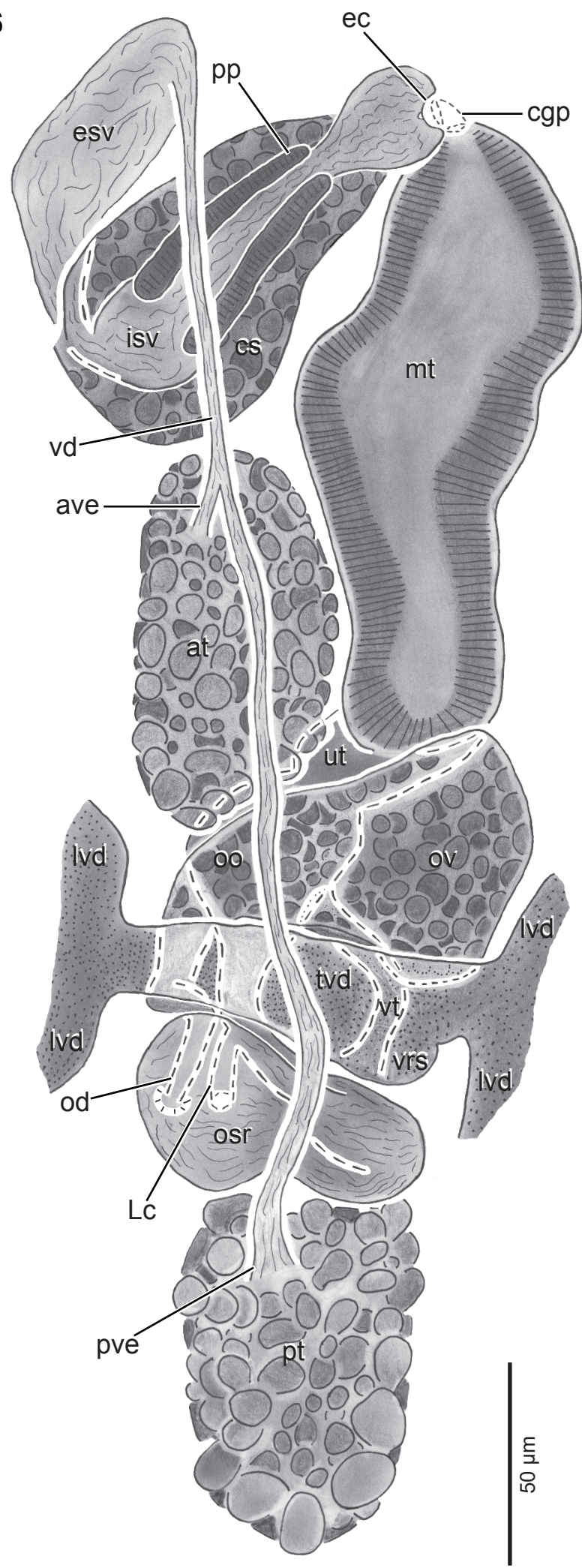

Figs. 5, 6. Coeuritrema platti sp. n. (Digenea: Schistosomatoidea) from viscera of Pelodiscus sinensis (Wiegmann) (Testudines: Trionychidae) from Vietnam, paratype (USNM 1411791). Fig. 5. Total view, ventrally. Fig. 6. Genitalia, ventral view. Abbreviations: at - anterior testis; ave - anterior trunk of vasa efferentia; cb - caecal bifurcation; cgp - common genital pore; cs - cirrus sac; $\mathrm{dc}$ - dextral caecum; ec - eversible cirrus; ep - excretory pore; esv - external seminal vesicle; ev - excretory vesicle; isv - internal seminal vesicle; Lc - Laurer's canal; lvd - lateral vitelline collecting duct; mt - metraterm; nc - nerve commissure; od - oviduct; oe - oesophagus; og - oesophageal gland; os - oral sucker; oo - ootype; osr - oviducal seminal receptacle; ov - ovary; ph - pharynx; $\mathrm{pp}$ - pars prostatica; $\mathrm{pt}$ - posterior testis; pve - posterior trunk of vasa efferentia; sc - sinistral caecum; tvd - transverse vitelline duct; ut - uterus; vd - vas deferens; vln - ventrolateral nerve chord; vlp - ventrolateral tegumental papillae; vr - vitellarium; vrs - vitelline reservoir; vs - ventral sucker; vt - vitelline duct. 
straight, $229-301(259 ; 6)$ long or $18-23 \%(21 \% ; 6)$ of body length, $10-15(13 ; 6)$ wide posterior to pharynx, with wall $7-10(9 ; 6)$ thick, $25-50(39 ; 6)$ maximum width or $(26 \% ; 6)$ of body width at caecal bifurcation, with wall $10-27(20 ; 6)$ thick, constricting to $13-18(16 ; 6)$ width or $8-16 \%(11 \% ; 6)$ of body width at caecal bifurcation, with wall 10-15 $(12 ; 6)$ thick; oesophageal gland 198-288 $(254 ; 6)$ long or $17-24 \%(21 \% ; 6)$ of body length and $0.9-1.1 \times(1.0 ; 6)$ oesophagus length, 75-125 $(104 ; 6)$ wide or $68-74 \%(71 \% ; 6)$ of body width at caecal bifurcation (Figs. 3, 5). Intestine bifurcating 235-365 (285; 6) or $17-27 \%(24 \% ; 6)$ of body length from anterior end; sinistral caecum $585-940(754 ; 6)$ long or $51-68 \%(62 \% ; 6)$ of body length, $35-45(39 ; 6)$ wide or $24-32 \%(27 \% ; 6)$ of body width at bifurcation, $13-30(20 ; 6)$ wide or $6-15 \%$ $(10 \% ; 6)$ of body width at ovary, $20-35(27 ; 6)$ wide or $12-19 \%(16 \% ; 6)$ of body width at termination; dextral caecum $585-940(743 ; 6)$ long or $52-67 \%(61 \% ; 6)$ of body length, $38-45(41 ; 6)$ wide or $24-36 \%(29 \% ; 6)$ of body width at bifurcation, $18-35(25 ; 6)$ wide or $10-18 \%$ $(12 \% ; 6)$ of body width at ovary, $18-35(27 ; 6)$ wide or $11-20 \%(16 \% ; 6)$ of body width at termination; caecal termination $92-140(120 ; 6)$ or $8-14 \%(10 \% ; 6)$ from posterior end.

Anterior testis follicular throughout, lacking lobes, $60-143(106 ; 6)$ long or $5-11 \%(9 \% ; 6)$ of body length, $50-95(71 ; 6)$ wide or $25-43 \%(35 \% ; 6)$ of body width at gonads; intertesticular space $30-103(74 ; 6)$ or $3-7 \%$ $(6 \% ; 6)$ of body length (Figs. 3-6). Posterior testis as anterior testis, $90-158(130 ; 6)$ long or $8-15 \%(11 \% ; 6)$ of body length, $55-125(89 ; 6)$ wide or $28-57 \%(44 \% ; 6)$ of body width at gonads; post-testicular space 293-335 $(310 ; 6)$ or $24-28 \%(27 \% ; 6)$ of body length from posterior end. Anterior trunk of vasa efferentia emanating from ventral surface of anterior testis, extending anteriad 10-35 $(26 ; 5)$ or $1-3 \%(2 \% ; 5)$ of body length from testis, $3(5)$ wide or $1-2 \%(1 \% ; 5)$ of body width (Figs. $4-6)$; posterior trunk of vasa efferentia emanating from ventral surface of posterior testis, extending anteriad 188-255 $(224 ; 5)$ or $17-21 \%(18 \% ; 5)$ of body length, $3-5(4 ; 5)$ wide or $1-3 \%$ $(2 \% ; 5)$ of body width, coalescing with anterior trunk of vasa efferentia ventral to anterior portion of anterior testis (Figs. 4-6).

Vas deferens $80-138(101 ; 5)$ long or $6-10 \%(8 \% ; 5)$ of body length, $3-5(4 ; 3)$ in maximum width, extending anteroventrally. External seminal vesicle $63-88(75 ; 5)$ long or $6 \%(5)$ of body length, $25-38(33 ; 5)$ wide, $1.9-2.5 \times$ $(2.3 ; 5)$ longer than wide, constricting posteriorly prior to internal seminal vesicle (Figs. 3-6); internal seminal vesicle $88-118(100 ; 5)$ long or $1.1-1.6 \times(1.4 ; 5)$ external seminal vesicle length, $13-35(23 ; 6)$ wide or $3.4-6.8 \times(4.4 ; 5)$ longer than wide or $52-92 \%(73 \% ; 5)$ of external seminal vesicle width (Figs. 3-6). Pars prostatica surrounding distal portion of internal seminal vesicle (Figs. 4, 6), 53-55 $(54 ; 3)$ long, $20-30(25 ; 3)$ wide or $1.8-2.7 \times(2.2 ; 3)$ longer than wide. Cirrus 5-8 (6;3) long, 3 (3) wide; cirrus sac enveloping most of internal seminal vesicle and entirety of pars prostatica, $78-128(100 ; 6)$ long or $7-9 \%(8 \% ; 6)$ of body length, $45-65(52 ; 6)$ wide or $22-30 \%(26 \% ; 6)$ of maximum body width, containing large putative secretory cells (Figs. 3-6).

Ovary triangular, with broadest portion in sinistral half of body, $160-320(246 ; 6)$ or $15-23 \%(20 \% ; 6)$ of body length from middle of ventral sucker, buttressing caeca, 35-115 $(84 ; 6)$ long or $3-9 \%(7 \% ; 6)$ of body length, $65-153(111$; 6) wide or $42-70 \%(54 \% ; 6)$ of body width, $1.1-1.9 \times(1.4$; 6) wider than long; post-ovarian space $425-510(458 ; 6)$ or $35-45 \%(28 \% ; 6)$ of body length (Figs. 3-6); oocytes uniform in size and basophilic throughout ovary (Figs. 4, 6). Oviduct extending posteriad, turning dorsally, proceeding sinistrad, turning dorsal again before extending posteriad for $35-105(61 ; 6)$ or $3-7 \%(5 \% ; 6)$ of body length, $5-10(8 ; 6)$ in maximum width, expanding laterally to form oviducal seminal receptacle; oviducal seminal receptacle extending sinistrad for $65-80(71 ; 3)$ or $63-78 \%(73 \% ; 3)$ of ovary width, $25-40(34 ; 5)$ in maximum width at origin or $11-20 \%(16 \% ; 5)$ of maximum body width, narrowing before turning dorsal, extending anterodextrad 78-80 (79; $3)$ or $6-7 \%(7 \% ; 3)$ of body length, $10-13(11 ; 3)$ in maximum width or $5-6 \%(5 \% ; 3)$ of body width (Figs. 3-6). Laurer's canal a narrow duct extending $15-25(21 ; 3)$ anterosinistrad from middle portion of oviduct, $5-8(6 ; 3)$ wide, opening dorsal and over proximal portion of oviducal seminal receptacle (Figs. 4, 6).

Vitellarium comprising a series of interconnected spheroid masses of follicles, distributing from level of caecal bifurcation to distal ends of caeca, lateral collecting ducts coalescing at level of posterior margin of ovary to form transverse vitelline duct; transverse vitelline duct ventral to ovary, 245-385 $(297 ; 3)$ or 21-27\% (24\%; 3$)$ of body length from middle of ventral sucker, lobed dorsally (Figs. 3-6); vitelline reservoir sac-like, ventral to oviducal seminal receptacle; vitelline duct extending anterodextrad and dorsal $30-58(47 ; 5)$ or $2-5 \%(4 \% ; 5)$ of body length before connecting with oviduct at ootype (Figs. 3-6). Ootype difficult to discern in gravid specimens, 25-40 $(32 ; 3)$ long, 33-43 $(38 ; 3)$ wide, dorsal to dextral half of ovary (Fig. 6).

Uterus comprising proximal portion and metraterm (Figs. 3-6), with a single egg in four of seven specimens; proximal portion of uterus extending anterosinistrad from ootype, $25-45(36 ; 3)$ long or $2-4 \%(3 \% 3)$ of body length, $23-38(29 ; 3)$ wide or $12-18 \%(14 \% ; 3)$ of maximum body width; metraterm extending anterosinistrad, 128-163 $(147 ; 5)$ long or $11-13 \%(12 \% ; 5)$ of body length, $45-63$ $(53 ; 3)$ wide or $13-32 \%(23 \% ; 3)$ of maximum body width, $2.8-5.8 \times(4.3 ; 3)$ proximal uterus length. Uterine egg ovoid, $80-95(86 ; 4)$ long or $6-8 \%(7 \% ; 4)$ of body length, $30-38(35 ; 4)$ wide or $14-17 \%(16 \% ; 4)$ of maximum body width, $2.3-2.7 \times(2.5 ; 4)$ longer than wide (Fig. 4). Common genital pore $63-138(103 ; 6)$ or $6-11 \%(8 \% ; 6)$ of body length posterior to middle of ventral sucker.

Excretory vesicle $120-180(165 ; 6)$ long or $12-17 \%$ $(14 \% ; 6)$ of body length, $38-90(74 ; 6)$ wide or $32-53 \%$ $(44 \% ; 6)$ of body width at caecal termination, with wall 5-15 (9; 4) thick (Figs. 3, 5).

Type and only known host: Chinese softshell turtle, Pelodiscus sinensis (Wiegmann) (Testudines: Trionychidae). 


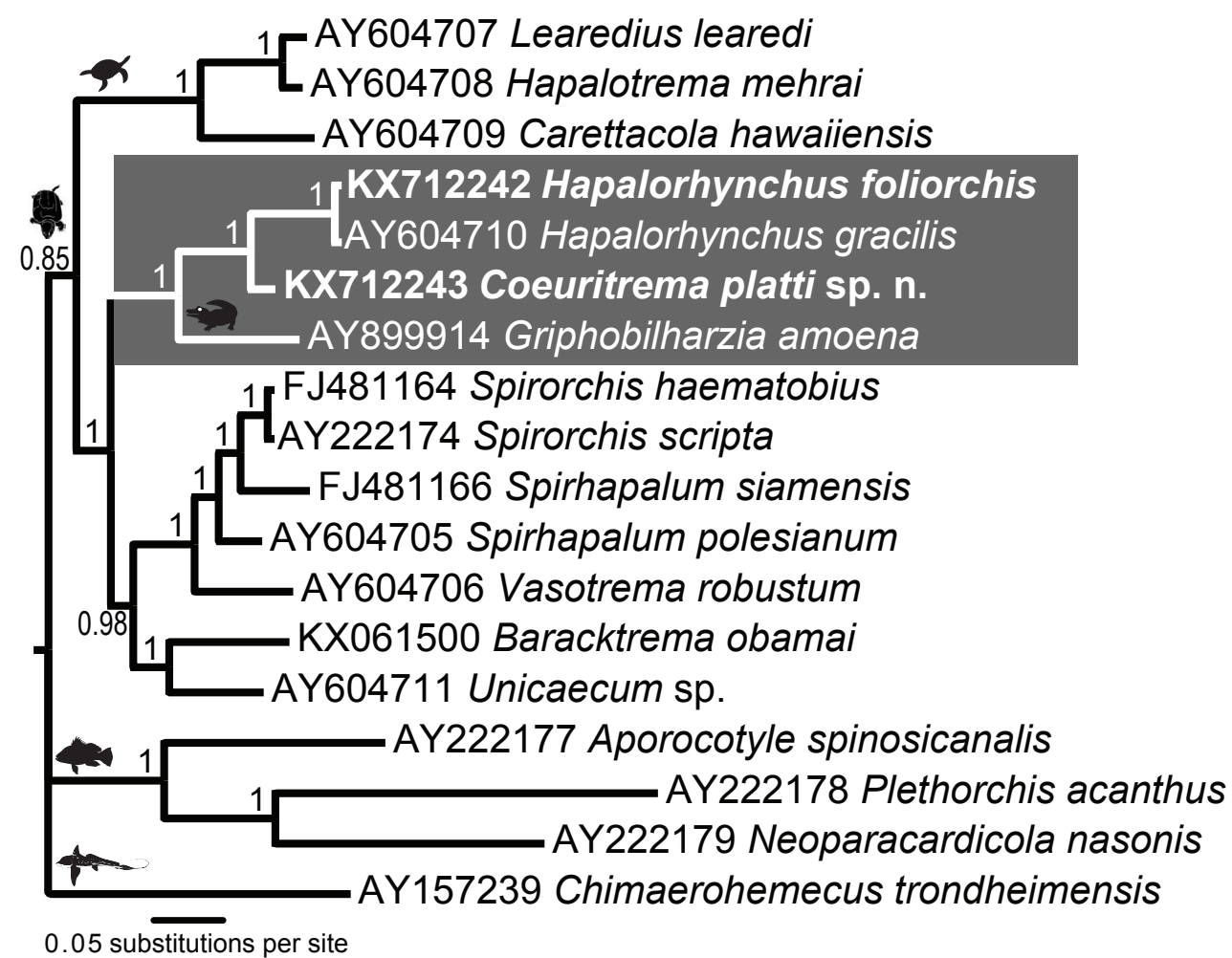

Fig. 7. Phylogenetic relationships of blood flukes reconstructed by Bayesian interference and based on partial D1-D3 domains of 28S from 18 taxa (13 TBFs, one crocodilian blood fluke, four fish blood flukes). Numbers aside tree nodes indicate posterior probability. Definitive hosts are indicated by icons aside tree nodes.

Type locality: Da Rang River Basin, Phu Yen Province, Vietnam.

Site in host: Heart, mesentery, lung.

Prevalence and intensity of infection: Two $(100 \%)$ P. sinensis from an aquaculture facility in the Da Rang River Basin had four and five specimens of C. platti. None of two P. sinensis collected from the Cai River Basin in Nha Trang yielded specimens.

Specimens/materials deposited: Holotype, United States National Museum (USNM) Coll. No. USNM 1411790; one paratype, United States National Museum (USNM) Coll. No. USNM 1411791; one paratype (Institute of Parasitology, Academy of Sciences of the Czech Republic, České Budějovice (IPCAS) Coll. No. D-727; GenBank Accession No. KX712243.

Material examined: Hapalorhynchus gracilis - American Museum of Natural History (AMNH) Coll. No. 125, holotype, one slide comprising one whole-mounted specimen, ex Chelydra serpentina from North Judson, Indiana (Stunkard 1922); AMNH 1269, paratypes, four slides (three of the four labeled Hapalorhychus gracilis) comprising four whole-mounted specimens, ex Chelydra serpentina from North Judson, Indiana (Stunkard 1922); Vasotrema attenuatum - AMNH 806, syntype, 17 slides comprising 25 whole-mounted specimens, ex Apalone ferox and A. spinifera from Florida and Indiana respectively (Stunkard 1928). Hapalorhynchus rugatus - HWML 21339.

Etymology: The specific epithet platti honours our friend and colleague Thomas R. Platt (Professor Emeritus, Saint Mary's College, Notre Dame, Indiana) for his extensive, sus- tained contributions to the taxonomy and systematics of turtle blood flukes.

Remarks. Coeuritrema platti is most similar to C. rugatus by the combination of having ventrolateral tegumental papillae restricted to the hindbody as well as a hindbody $<1.6 \times$ forebody width. The new species is most easily distinguished from $C$. rugatus by having small ventrolateral tegumental papillae ( $35 \mu \mathrm{m}$ maximum base width), testes without deep lobes, and a Laurer's canal pore opening posterior to the vitelline reservoir and dorsal to the oviducal seminal receptacle. Coeuritrema rugatus has large ventrolateral tegumental papillae $(53 \mu \mathrm{m}$ maximum base width), deeply lobed testes, and a Laurer's canal pore opening anterior to the vitelline reservoir and oviducal seminal receptacle. Coeuritrema platti differs from C. lyssimus by having a narrow hindbody $(1.1-1.4 \times$ forebody width), ventrolateral tegumental papillae restricted to the hindbody, a short cirrus sac extending $7-9 \%$ of body length, a transverse ovary buttressing the caeca, a short, wholly pre-ovarian metraterm (11-13\% of body length), and a submarginal genital pore. Coeuritrema lyssimus has a wide hindbody $(1.7 \times$ forebody width), ventrolateral tegumental papillae distributing from the oral sucker to the excretory pore, a long cirrus sac extending 10-13\% of body length, a sinistral ovary that does not buttress the caeca, a metraterm that begins lateral to the ovary and extends anteriad $17-18 \%$ of the body length, and a marginal genital pore. 


\section{Molecular phylogenetic results}

Fig. 7

The results of our phylogenetic analysis of the D1-D3 domains of the nuclear large subunit ribosomal DNA (28S) produced three clades: the chondrichthyan blood fluke Chimaerohemecus trondheimensis van der Land, 1967, the blood flukes of bony fishes (Aporocotyle spinosicanalis Williams, 1958, Plethorchis acanthus Martin, 1975 and Neoparacardicola nasonis Yamaguti, 1970), and the TBFs, including the enigmatic crocodilian blood fluke Griphobilharzia amoena (Fig. 7). Within TBFs, and as already reported elsewhere (Snyder 2004, Orélis-Ribeiro et al. 2014, Roberts et al. 2016), the marine (Carettacola Manter et Larson, 1950 clade) and freshwater TBFs clustered separately and with high nodal support. Within the freshwater TBFs, we recovered separate Hapalorhynchus and Spirorchis clades. The new species was sister to Hapalorhynchus spp., and that clade was sister to G. amoena. The phylogenetic distance (branch lengths) within the Hapalorhynchus clade supported our conclusions from morphology in that Coeuritrema is distinctive from other accepted TBF genera. Additional sequences from Enterohaematotrema and Cardiotrema are required to test monophyly of Coeuritrematinae (see above).

\section{DISCUSSION}

Our morphological descriptions and comparisons coupled with our molecular phylogenetic results indicated that Coeuritrema shares a recent common ancestor with Hapalorhynchus (Fig. 7). However, definitive host ecology, phylogenetic affiliation and geographic distribution do not explain the observed sister-taxa relationships. In specific, both $H$. gracilis (type species) and $H$. foliorchis infect the common snapping turtle, Chelydra serpentina (Testudines: Chelydridae), whereas $C$. platti infects $P$. sinensis (Trionychidae). Chelydra serpentina and $P$. sinensis are assigned to different families and those families are not phylogenetically closely related (Guillon et al. 2012, Crawford et al. 2015). Moreover, none of these TBFs nor their turtle hosts have overlapping geographic distributions: $\mathrm{H}$. gracilis and $H$. foliorchis range in North America only, whereas $C$. platti ranges in Vietnam only (Table 1). Excluding definitive host ancestry and biogeography, we predict that knowledge of the life cycles of species of Hapalorhynchus, Coeuritrema and Griphobilharzia could help explain the observed topology. Unfortunately, no life cycle is known for any species of the Hapalorhynchus clade, precluding a deeper discussion of this matter herein. Orélis-Ribeiro et al. (2014) concluded that blood fluke clades can be identified by their molluscan intermediate hosts, with marine flukes infecting bivalves plus freshwater and estuarine flukes infecting snails. Additional morphological, life history and sequence data sourced from the other 17 accepted species of Hapalorhynchus and additional species of
Coeuritrema are required to further test the phylogenetic pattern we recovered herein (Fig. 7).

Especially noteworthy herein is the sister relationship between Griphobilharzia amoena and the Coeuritrema + Hapalorhynchus clade. Morphologically, G. amoena appears as a schistosome (although having a single testis and lacking fused caeca), with markedly distinctive morphological features that do not intuitively align it with Coeuritrema nor Hapalorhynchus or other TBFs for that matter. Griphobilharzia Platt et Blair, 1991 resembles schistosomatids by dioecity and having a ventral sucker (Platt et al. 1991, 2013). Further, by having a well-developed gynaecophoric canal (see Khalil 2002), it resembles several genera of Schistosomatinae Stiles et Hassall, 1898: Schistosoma Weinland, 1858, Ornithobilharzia Odhner, 1912, Austrobilharzia Johnston, 1917, Macrobilharzia Travassos, 1922, Schistosomatium Tanabe, 1923, Heterobilharzia Price, 1929, Bivitellobilharzia Vogel et Minning, 1940 and Orientobilharzia Dutt et Srivastava, 1955.

The stark discordance between comparative morphology and gene sequence analysis reiterate the need for additional molecular gene sequence data from specimens identified as G. amoena. Griphobilharzia amoena differs from all other TBFs by being dioecious and by maturing in the freshwater crocodile, Crocodylus johnstoni Krefft. Griphobilharzia resembles all TBFs, except Baracktrema (single caecum), Neospirorchis Price, 1934 (fused caeca) and Unicaecum Stunkard, 1925 (single caecum), by having two non-fused caeca. Griphobilharzia resembles $\mathrm{Ba}$ racktrema, Neospirorchis, Unicaecum, Uterotrema Platt et Pichelin, 1994, and Vasotrema by having a single testis.

Regarding the systematics of blood flukes sensu lato, for now, this clade as well as the marine TBF clade (Carettacola, Hapalotrema Looss, 1899 and Learedius Price, 1934), must continue to remain in systematic limbo, without a familial assignment (Looss 1899, Price 1934, Manter and Larson 1950, Orélis-Ribeiro et al. 2014, Roberts et al. 2016).

Acknowledgements. We thank Dang Nguyen Anh Tuan and Tran Quang Sang (Nha Trang University) as well as Matthew R. Womble (National Oceanic and Atmospheric Administration, Washington, DC) for helping collect turtles and blood flukes in Vietnam, and Gabor Racz (HWML) and Estefania Rodriguez (AMNH) for loaning museum specimens. The present study is a contribution of the Southeastern Cooperative Fish Parasite and Disease Project (Auburn University) and was supported in part by a grant from the York International Scholars Program (Auburn University) awarded to JRR, ROR and SAB and by the National Science Foundation Division of Environmental Biology via grant nos. 1112729,1051106 (also with KHM) and 1048523 awarded to SAB. We thank and are indebted to Tom Platt for his donation of his library and helminthological collection to SAB, which made possible the revisionary systematics work presented herein and that which is forthcoming. 


\section{REFERENCES}

Alves R.R.N., Vieira W.L.S., Santana G.G. 2008: Reptiles used in traditional folk medicine: conservation implications. Biodivers. Conserv. 17: 2037-2049.

Belous E.V. 1963: [Helminth fauna of water turtles, Amyda sinensis, of the Far East.] Helminthologia 4: 79-99. (In Russian.)

Bourgat R. 1990: Extension taxonomique et biogeographique du genre Hapalorhynchus (Trematoda, Spirorchiidae). Bull. Soc. Fr. Parasitol. 8: 289-294.

Brooks D.R., Mayes M.A. 1975: Platyhelminths of Nebraska turtles with descriptions of two new species of spirorchiids (Trematoda: Spirorchiidae). J. Parasitol. 61: 403-406.

Brooks D.R., Mayes M.A. 1976: Telorchis gutturosi sp. n. (Trematoda: Telorchiidae) from Graptemys pseudogeographica Gray in Nebraska, with reports of additional species of trematodes from Nebraska turtles. J. Parasitol. 62: 901-905.

Brooks D.R., Sullivan J.J. 1981. Hapalorhynchus rugatus sp. nov. (Digenea: Spirorchidae) from a Malaysian freshwater turtle. Can. J. Zool. 59: 1335-1338.

Bullard S.A., Jensen K. 2008. Blood flukes (Digenea: Aporocotylidae) of stingrays (Myliobatiformes: Dasyatidae): Orchispirium heterovitellatum from Himantura imbricata in the Bay of Bengal and a new genus and species from Dasyatis sabina in the northern Gulf of Mexico. J. Parasitol. 94: 1311-1321.

Bullard S.A., Jensen K., Overstreet R.M. 2009. Historical account of the two family-group names in use for the single accepted family comprising the "fish blood flukes." Acta Parasitol. 54: 78-84.

Bullard S.A., Overstreet R.M. 2006. Psettarium anthicum sp. n. (Digenea: Sanguinicolidae) from the heart of cobia Rachycentron canadum (Rachycentridae) in the northern Gulf of Mexico. Folia Parasitol. 53: 117-124.

Bullard S.A., Overstreet R.M. 2008. Chapter 14: Digeneans as enemies of fishes. In: J. Eiras, H. Segner, T. Wahil and B.G. Kapoor (Eds.), Fish Diseases. Science Publishers, New Hamphsire, pp. 817-976.

Bullard S.A., Overstreet R.M., Carlson, J.K. 2006. Selachohemecus benzi n. sp. (Digenea: Sanguinicolidae) from the blacktip shark Carcharhinus limbatus in the northern Gulf of Mexico. Syst. Parasitol. 63: 143-154.

Byrd E.E. 1939: Studies on the blood flukes of the family Spirorchidae. Part II. Revision of the family and description of new species. J. Tenn. Acad. Sci. 14: 116-161.

Castresana J. 2000: Selection of conserved blocks from multiple alignments for their use in phylogenetic analysis. Mol. Biol. Evol. 17: 540-552.

Crawford N.G., Parham J.F., Sellas A.B., Faircloth B.C., Glenn T.C., Papenfuss T.J., Henderson J.B., Hansen M.H., Simison W.B. 2015: A phylogenomic analysis of turtles. Mol. Phylogenet. Evol. 83: 250-257.

Darriba D., Taboada G.L., Doallo R., Posada D. 2012: jModelTest 2: more models, new heuristics and parallel computing. Nat. Methods 9: 772 .

van Dijk P.P., Iverson J.B., Rhodin A.G. J., Shaffer H.B., Bour R. 2014: Turtles of the world: annotated checklist of taxonomy, synonymy, distribution with maps, and conservation status. Seventh Edition. Chel. Res. Monogr. 5: 329-479.

van Dijk P.P., Stuart B.L., Rhodin A.G.L. 2000: Asian Turtle trade. Proceedings of a Workshop on Conservation and Trade of Freshwater Turtles and Tortoises in Asia, Phnom Penh, Cambodia, 1-4 December 1999. Chelonian Research Foundation, Lunenburg, Massachusetts, $164 \mathrm{pp}$.

Dwivedi M.P. 1967: Contribution to the family Spirorchiidae Stunkard, 1921 (Digenea: Trematoda). Ind. J. Helminthol. 19: $1-14$.

Fritz U., Gong S., Auer M., Kuchling G., Schneeweif, HundSDÖRFER A. K. 2010: The world's economically most important chelonians represent a species complex (Testudines: Trionychidae: Pelodiscus). Org. Divers. Evol. 10: 227-242.
Guillon J., Guéry L., Hulin V., Girondot M. 2012: A large phylogeny of turtles (Testudines) using molecular data. Contr. Zool. 81: 147-158.

Guindon S., Gascuel O. 2003: A simple, fast and accurate method to estimate large phylogenies by maximum-likelihood. Syst. Biol. 52: 696-704.

Haitao S., Parham J.F., Zhiyong F., Meiling H., Feng Y. 2008. Evidence for the massive scale of turtle farming in China. Oryx 42: 147-150.

Huelsenbeck J.P., Ronquist F. 2005: Bayesian analysis of molecular evolution using MrBayes. In: R. Nielsen (Ed.), Statistical Methods in Molecular Evolution. Springer Verlag, New York, pp. 183-232.

Huelsenbeck J.P., Ronquist F., Nielsen R., Bollback J.P. 2001: Bayesian inference of phylogeny and its impact on evolutionary biology. Science 294: 2310-2314.

Hughes R.C., Higginbotham J.W., Clary J.W. 1942: The trematodes of reptiles, Part I. Am. Mid. Nat. 27: 109-134.

International Commission ON Zoological Nomenclature 2000: International Code of Zoological Nomenclature (ICZN), Fourth Edition, The Natural History Museum, London, 306 pp.

КАтон K., Тон. H. 2010: Parallelization of the MAFFT multiple sequence alignment program. Bioinformatics 26: 1899-1900.

KhaliL L.F. 2002: Family Schistosomatidae Stiles \& Hassall, 1898. In: D.I. Gibson, A.J. Jones and R.A. Bray (Eds.), Keys to the Trematoda, Vol. 1. CABI, Wallingford, pp. 419-432.

Lamothe-Argumedo R. 1978: Tremátodes de reptiles 1. Description de una especie nueve de la familia Spirorchidae, parásita de Kinosternon leucostomum de Villahermosa, Tabasco, México. An. Inst. Biol. Univ. Nal. Autón. Méx. 49: 19-24.

Lockyer A.E., Olson P.D., Ostergaard P., Rollinson D., Johnston D.A., Attwood S.W., Southgate V.R., Horák P., Snyder S.D., Le T. H., Agatsuma T., McManus D.P., Carmichael A.C., Naem S., Littlewood D.T.J. 2003: The phylogeny of the Schistosomatidae based on three genes with emphasis on the interrelationships of Schistosoma Weinland, 1858. Parasitology 126: 203-224.

LofTIN H. 1960: An annotated check-list of trematodes and cestodes and their vertebrate hosts from northwest Florida. Q. J. Florida Acad. Sci. 23: 302-314.

Looss A. 1899: Weitere Beiträge zur kenntnis der Trematoden-Fauna Aegyptens, zugleich versuch einer natürlichen Gleiderung des genus Distomum Retzius. Zool. Jahrb. 12: 521-784.

LuHMAN M. 1935: Two new trematodes from the loggerhead turtle (Caretta caretta). J. Parasitol. 21: 274-276.

MANTER H.W., LARSON M.I. 1950: Two new blood flukes from a marine turtle, Caretta caretta. J. Parasitol. 36: 595-599.

Menra H.R. 1933: New blood flukes of the family Spirorchidae Stunkard from Indian fresh-water tortoises with discussion on the synonymy of certain genera and the relationships of the families of blood flukes. Part I. Bull. Acad. Sci. United Prov. Agra Oudh, India 2: 203-225

Menra H.R. 1934: New blood flukes of the family Spirorchidae Stunkard from Indian fresh-water tortoises with discussion on the synonymy of certain genera and the relationships of the families of blood flukes. Part II. Bull. Acad. Sci. United Prov. Agra Oudh, India 3: 169-196.

Menra H.R. 1939: New blood flukes of the family Spirorchidae Stunkard (Trematoda) from the marine turtle Chelone mydas of the Arabian Sea with observations on the synonymity of certain genera and classification of the family. Proc. Nat. Acad. Sci. India 9: $155-167$.

Mehra H.R. 1940: A new distome Enterohaematotrema n. g. and a new blood fluke Hemiorchis bengalensis n. sp. belonging to the family Spirorchidae Stunkard, and a new species of the genus Dendritobilharzia Skrjabin and Zakharow belonging to the family Schistosomatidae Poche, with remarks on the evolution 
of the blood flukes. Proc. Nat. Acad. Sci. India. Section B, Biol Sci. 10: 100-118.

Menrotra V. 1973: Digenea from some reptile hosts in India, Part II (in continuation with Abstract No. 286 in Part III of the Proceedings). Proc. Sixtieth Ind. Sci. Cong. 4: 46-47.

Miller M.A., Pfeiffer W., Schwartz T. 2010. Creating the CIPRES Science Gateway for inference of large phylogenetic trees. Proceedings of the Gateway Computing Environments Workshop (GCE). New Orleans, Louisiana, 8 pp.

Orélis-Ribeiro R., Arias C.R., Halanych K.M., Cribb T.H., BULLARD S.A. 2014: Diversity and ancestry of flatworms infecting blood of nontetrapod craniates "fishes." Adv. Parasitol. 85: $1-64$.

OshmaRIN P.G. 1971: [A new species of trematode from the blood vessels of Chelonia.] In: Parazity Zhivotnykhi Rasternii Dal'nego Vostoka, Dal'nevostochnoe Knizhnoe Izdatel'stvo, Vladivostok, pp. 142-143. (In Russian.)

Platt T.R. 1988: Hapalorhychus brooksi sp. n. (Trematoda: Spirorchiidae) from the snapping turtle (Chelydra serpentina), with notes on $H$. gracilis and H. stunkardi. Proc. Helminthol Soc. Wash. 55: 317-323.

Platt T.R. 1993: Taxonomic revision of Spirorchis MacCallum, 1919 (Digenea: Spirorchidae). J. Parasitol. 79: 337-346.

Platt T.R. 1998: Redescription of Hapalotrema mistroides (Monticelli, 1896) and Hapalotrema synorchis Luhman, 1935 (Digenea: Spirorchidae), with comments on other species in the genus. J. Parasitol. 84: 594-600.

Platt T.R. 2002: Family Spirorchiidae Stunkard, 1921. In: D.I. Gibson, A.J. Jones and R.A. Bray (Eds.), Keys to the Trematoda, Vol. 1. CABI, Wallingford, 453-467 pp.

Platt T.R., Blair D., Purdie J., Melville L. 1991: Griphobilharzia amoena $\mathrm{n}$. gen., n. sp. (Digenea: Schistosomatidae), a parasite of the freshwater crocodile Crocodylus johnstoni (Reptilia: Crocodylia) from Australia, with the erection of a new subfamily, Griphobilharzinae. J. Parasitol. 77: 65-68.

Platt T.R., Hoberg E.P., Chisholm L.A. 2013: On the morphology and taxonomy of Griphobilharzia amoena Platt and Blair, 1991 (Schistosomatoidea), a dioecious digenetic trematode parasite of the freshwater crocodile, Crocodylus johnstoni, in Australia. J. Parasitol. 99: 888-891.

Platt T.R., Prestwood A.K. 1990: Deposition of type and voucher material from the helminthological collection of Elon E. Byrd. Syst. Parasitol. 16: 27-34.

Platt T.R., Sharma R.S.K. 2012: Two new species of Hapalorhynchus (Digenea: Spirorchiidae) from freshwater turtles (Testudines: Geoemydidae) in Malaysia. Comp. Parasitol. 79: 202-207.

Platt T.R., SNYDER S.D. 2007: Redescription of Hapalorhynchus reelfooti Byrd, 1939 (Digenea: Spirorchiidae) from Sternotherus odoratus (Latreille, 1801). Comp. Parasitol. 74: 31-34.

Posada D., Buckley T.R. 2004: Model selection and model averaging in phylogenetics: advantages of Akaike Information Criterion and Bayesian approaches over likelihood ratio tests. Syst. Biol. 53: 793-808.

Price E.W. 1934: New genera and species of blood flukes from a marine turtle, with a key to the genera of the family Spirorchidae. J. Wash. Acad. Sci. 24: 132-141.

Rambaut A. 2009: FigTree v1.2.3, Institute of Evolutionary Biology, Univ. of Edinburgh. Available at: http://tree.bio.ed.ac.uk/ software/figtree.

Rambaut A., Drummond A.J. 2009: Tracer Version 1.5. Program. Available at: http://beast.bio.ed.ac.uk/Tracer/.

Roberts J.R., Platt T.R., Orélis-Ribeiro R., Bullard S.A. 2016: New genus of blood fluke (Digenea: Schistosomatoidea) from Malaysian freshwater turtles (Geoemydidae) and its phylogenetic position within Schistosomatoidea. J. Parasitol. 102: 451-462.

Rohde K., Lee S. K., Lim H.W. 1968: Ueber drei malayische Trematoden. Ann. Parasitol. Hum. Comp. 43: 33-43.

Ronquist F., Huelsenbeck J.P. 2003: MRBAYES 3: Bayesian phylogenetic inference under mixed models. Bioinformatics. 19: 1572-1574.

ShrivastaVA P.S. 1959: Cercaria of Enterohaematotrema palaeorticum Mehra, 1940. Proc. First All-India Cong, Zool. 2: 460-465.

Sмiтн J.W. 1997a: The blood flukes (Digenea: Sanguinicolidae and Spirorchidae) of cold-blooded vertebrates: Part I. A review of the literature published since 1971, and bibliography. Helminthol. Abstr. 66: 255-294

Sмiтн J.W. 1997b: The blood flukes (Digenea: Sanguinicolidae and Spirorchidae) of cold-blooded vertebrates: Part II. Appendix I: Comprehensive parasite-host list; Appendix II: Comprehensive host-parasite list. Helminthol. Abstr. 66: 329-344.

SNYDER S.D. 2004. Phylogeny and paraphyly among tetrapod blood flukes (Digenea: Schistosomatidae and Spirorchiidae). Int. J. Parasitol. 34: 1385-1392.

Stuckas H., Fritz U. 2011: Identity of Pelodiscus sinensis revealed by DNA sequences of an approximately 180-year-old type specimen and a taxonomic reappraisal of Pelodiscus species (Testudines: Trionychidae). J. Zool. Syst. Evol. Res. 49: 335-339.

StUNKARD H.W. 1922: Two new genera of North American blood flukes. Am. Mus. Novit. 39: 1-8.

STUNKARD H.W. 1926: A new trematode Vasotrema amydae n. g., n. sp., from the vascular system of the soft-shelled turtle, Amyda. Anat. Rec. 34: 165.

Stunkard H.W. 1928: Observations nouvelles sur les trématodes sanguicoles du genre Vasotrema (Spirorchidae) avec description des deux espèces nouvelles. Ann. Parasitol. Hum. Comp. 6: $303-320$.

TAKeuti E. 1942. New blood flukes of the family Spirorchidae from Japanese fresh-water tortoise and marine turtles. Jpn. J. Med. Sci. VI, Bact. Parasitol. 2: 161-174.

Tamura K.D., Peterson D., Peterson N., Stecher G., Nei M., Kumar S. 2011: MEGA5: molecular evolutionary genetics analysis using maximum likelihood, evolutionary distance, and maximum parsimony methods. Mol. Biol. Evol. 28: 2731-2739.

Tandon V., Gupta N.K. 1982: On some blood flukes (Spirorchiidae: Coeuritrematinae) from freshwater chelonians in India. Proc. Ind. Acad. Sci. An. Sci. 91: 275-282.

Tandon V., Gupta, N.K. 1985: On the blood fluke, Cardiotrema roparensis Mehrotra, 1973 and validity of C. longivesticulata Dwivedi, 1967 (Spirorchiidae: Coeuritrematinae). Res. Bull. (Sci.) Panjab Univ. 36: 331-334.

Thapar G. S. 1933. A new blood fluke from an Indian tortoise, Trionyx gangeticus. J. Helminthol. 11: 163-168.

TKaCh V., SNyder S.D., Vaughan J.A. 2009: A new species of blood fluke (Digenea: Spirorchiidae) from the Malayan box turtle, Cuora amboinensis (Cryptodira: Geoemydidae) in Thailand. J. Parasitol. 95: 743-746.

WALL L.D. 1951: The life history of Vasotrema robustum (Stunkard, 1928), Trematoda: Spirorchiidae. Trans. Am. Microsc. Soc. 70: 173-184.

Yamaguti S. 1958: Systema Helminthum. Volume 1, The Digenetic Trematodes of Vertebrates, Part I. Interscience Publishers, New York, 979 pp.

Yamaguti S. 1971: Synopsis of Digenetic Trematodes of Vertebrates, Vol. I. Keigaku Publishing Company, Tokyo, 1074 pp.

Cite this article as: Roberts J.R., Orélis-Ribeiro R., Dang B.T., Halanych K.M., Bullard S.A. 2016: Blood flukes of Asiatic softshell turtles: revision of Coeuritrema Mehra, 1933 (Digenea: Schistosomatoidea) and a new species infecting Chinese softshell turtles, Pelodiscus sinensis, (Trionychidae) from Vietnam. Folia Parasitol. 63: 031. 